\title{
ELECTRE METHODS WITH INTERACTION BETWEEN CRITERIA: AN EXTENSION OF THE CONCORDANCE INDEX
}

\author{
José Rui Figueira *, Salvatore Greco ${ }^{\dagger}$, Bernard Roy ${ }^{\ddagger}$
}

April 30, 2009

\footnotetext{
${ }^{*}$ CEG-IST, Center for Management Studies, Instituto Superior Técnico, Technical University of Lisbon, TagusPark, Av. Cavaco Silva, 2780 990 Porto Salvo, Portugal. Phone: +351 2142332 99, Fax: +351 21423 35 68, E-mail: figueira@ist.utl.pt (Also Associate Researcher at LAMSADE, Université Paris-Dauphine, Place du Maréchal De Lattre de Tassigny, 75775 Paris Cedex 16, France)

${ }^{\dagger}$ Faculty of Economics, The University of Catania, Corso Italia, 55, 95129 Catania, Italy. Phone: +39 095375344 (Ext: 202 ), Fax: +39 095 3705 74, E-mail: salgreco@unict.it

‡LAMSADE, Université Paris-Dauphine, Place du Maréchal De Lattre de Tassigny, 75775 Paris Cedex 16, France. Phone: +33 144054434 , Fax: +331440540 91, E-mail: roy@lamsade.dauphine.fr
} 


\title{
ELECTRE METHODS WITH INTERACTION \\ BETWEEN CRITERIA: AN EXTENSION \\ OF THE CONCORDANCE INDEX
}

\begin{abstract}
This paper presents an extension of the comprehensive (overall) concordance index of ELECTRE methods, which takes the interaction between criteria into account. In real-world decision-aiding situations, it is reasonable to consider only the interaction between a small number of criterion pairs. Three types of interaction have been considered: mutual strengthening, mutual weakening, and antagonistic. The new concordance index correctly takes into account such types of interactions, by imposing such conditions as boundary, monotonicity, and continuity. We demonstrate that the generalized index is able to take the three types of interaction, or dependencies, between criteria into account satisfactorily, first using quasi criteria and then using pseudo criteria. We also examine the links between the new concordance index and the Choquet integral
\end{abstract}

Key-words: Multicriteria analysis, Outranking methods, Interaction between criteria, Choquet integral.

\section{Introduction}

In this article, we are particularly interested in those decision-aiding situations that can be supported using an ELECTREtype method (cf. Figueira et al., 2005 and Roy and Vanderpooten, 1997). This kind of situation implies that a coherent family $F$ of $n$ criteria has previously been built (cf. Roy and Bouyssou, 1993 and Roy, 1996).

An important advantage of using outranking methods (e.g., ELECTRE methods) is that they are able to take purely ordinal scales into account (Martel and Roy, 2006), without needing to convert the original scales into abstract ones with an arbitrary imposed range, thus maintaining the original concrete verbal meaning (for another methodology considering purely ordinal scales, see Greco et al., 2001). Such conversions are used in many multi-criteria methods - for example, AHP (Saaty, 2005), MACBETH (Bana e Costa and Vansnick, 1994; Bana e Costa et al., 2005), MAUT (Keeney and Raiffa, 1976), SMART (Edwards, 1977; Von Winterfeldt and Edwards, 1986), TOPSIS (Hwang and Yoon, 1981) - as well as in methods based on fuzzy integrals (Grabisch, 1996; Grabisch and Labreuche, 2005). A second advantage is that indifference and preference thresholds can be taken into account when modeling the imperfect knowledge of data, which is impossible in the previous mentioned methods.

When using an ELECTRE-type method ( whatever a particular version of the method is considered), the criteria family $F$ must be designed so that there is no significant interaction between any criterion pairs. By definition, we say that there is significant interaction between two criteria if links (whatever their nature) exist between these criteria that must be taken into account to support the validity, credibility, or intensity of the comprehensive preference relationships built by the model (based on $F$ ) to clarify the decision. In fact, fuzzy integral-based methods were introduced in decision aiding to allow such interactions to be taken into account. This article proposes and extends ELECTRE methods that allow certain types of interactions to be taken into account very concretely. Specifically, this paper extends the notion of concordance, as it has been defined for ELECTRE methods, except for ELECTRE IV (see Figueira et al., 2005), to three particular types of interaction, designated here as mutual strengthening, mutual weakening, and antagonistic.

The rest of this paper is organized as follows. Section 2 provides two examples to clarify the reader's understanding of the three types of interactions that can occur in real-world decision-aiding situations. Section 3 introduces the fundamental concepts, definitions, and notation, and reviews the general notions of comprehensive concordance index as well as its fundamental properties. Section 4 defines the three types of interaction considered in this paper, as well as how the decision-maker (DM) can assign numerical values to the parameters characterizing these interactions. Section 5 presents an extension of the concordance index, starting with the simplest case in which only quasi criteria are considered, and then moving towards the more complex case in which pseudo-criteria are considered. In Section 6, the examples provided in Section 2 are discussed in order to assess the contribution of our extension. Section 7 compares our method with Choquet integral methods to evaluate how the two approaches take the interactions between criteria into account. The last section offers our conclusions and lines for future research. 


\section{Illustrative Examples}

This section provides two examples in order to clarify the effects of the different interactions dealt with in this paper. These effects generate additional information that must be taken into account in the concordance indices. (Section 6 offers a more detailed discussion of how such additional information can be modeled.) In the criterion descriptions, [min] is assigned to the criteria to be minimized and $[\max ]$ to the criteria to be maximized.

\subsection{Choosing a site for a new hotel construction project}

In this example, a site must be selected for a new hotel, which belongs to a multinational group, in a city where the group is not yet established. Suppose that a consulting company (henceforth, called the analyst) was asked to support that decision-aiding process of the Chief Executive Officer (CEO) of this group (henceforth, called DM), and that this analyst and the decision-maker's representative (henceforth, called DMR) decided to use an ELECTRE-type method. To this end, a family of five criteria $\left(g_{1}-g_{5}\right)$ is built:

$g_{1}$ : land purchasing and construction costs (investment costs) [min];

$g_{2}$ : annual operating costs (annual costs) [min];

$g_{3}$ : personnel recruitment possibilities (recruitment) [max];

$g_{4}$ : target client perceptions of the city district (image) [max];

$g_{5}$ : facility of access for the target clients (access) [max].

Indifference and preference thresholds (see Section 3) are associated with each one of these criteria. For the first two criteria, which are quantitative, these thresholds model the "approximate" character of the financial evaluations, and for the three other criteria, the unavoidable arbitrariness of the value due to the subjectivity of purely ordinal evaluations. These criteria do not have the same importance for the DMR. In order to represent these differences, intrinsic relative weights $k_{j}, j=1, \ldots, 5$, are associated to the corresponding criteria in the aggregation procedure, using the $\mathrm{SRF}$ (acronym of Simos-Roy-Figueira) technique and software by Figueira and Roy (2002). When considering two criteria in SRF, the value of each weight is fixed without taking into account the impact that the other criterion weight can have independently of whether or not belongs to the concordant coalition; in other words, all the possible interactions between criteria are abstracted.

The following tables given information that would allow the DMR to see how the weights intervene in the comparison of two sites.

a) The comparison of site $a$ with sites $b, c$, and $d$, in terms of the two financial criteria $g_{1}$ and $g_{2}$, is shown in Table 1 .

\begin{tabular}{|l|c|c|c|}
\hline & $b$ & $c$ & $d$ \\
\hline$g_{1}$ & $a$ is better than $b$ & $a$ is worse than $c$ & $a$ is better than $d$ \\
\hline$g_{2}$ & $a$ is worse than $b$ & $a$ is better than $c$ & $a$ is better than $d$ \\
\hline
\end{tabular}

Table 1: Comparison of site $a$ with sites $b, c$, and $d$ with respect to the financial criteria

According to the classic definition of the concordance index (see Section 3), the role that criteria $g_{1}$ and $g_{2}$ should have for supporting the answer to the assertion " $a$ is at least as good as $b$ (or $c$ or $d$ )" is characterized by the following weights,

$k_{1}$ in the comparison with $b$,

$k_{2}$ in the comparison with $c$,

$k_{1}+k_{2}$ in the comparison with $d$. 
Given the information presented in Table 1, the DMR considers the weights $k_{1}$ and $k_{2}$ assigned to criteria $g_{1}$ and $g_{2}$ appropriate, when only one criterion, $g_{1}$ or $g_{2}$, supports a decision that one action is better than another one. However, he/she judges that the sum $k_{1}+k_{2}$ is not sufficient to characterize the role of this criteria pair when both supports the decision that one action is better than another one, because in this case each criterion is strengthened by the other given the degree of complementarity between them. The comparisons provided by the DMR about actions $a, b, c$, and $d$ express his/her conviction that, if one action is better than another one with respect to criteria $g_{1}$ and $g_{2}$ conjointly, it would be interesting to be able to take this mutual strengthening effect into account. As this reasoning shows, the classic concordance index is not able to take such a mutual strengthening effect into account (for an illustrative example see Section 6). This effect can be taken into account by increasing the weights $k_{1}$ and $k_{2}$ for the criteria $g_{1}$ and $g_{2}$, respectively in the concordance index of the assertion " $a$ is at least as good as $d$ ", when both criteria intervene conjointly to make the assertion valid. In the following sections, the amount that must be added to $k_{1}+k_{2}$ to model this mutual strengthening effect is denoted $k_{12}=k_{21}$.

$b$ ) The comparisons of site $a$ with sites $b, c$, and $e$ in terms of the two purely ordinal criteria, $g_{4}$ and $g_{5}$, are presented in Table 2.

\begin{tabular}{|l|c|c|c|}
\hline & $b$ & $c$ & $e$ \\
\hline$g_{4}$ & $a$ is better than $b$ & $a$ is worse than $c$ & $a$ is better than $e$ \\
\hline$g_{5}$ & $a$ is worse than $b$ & $a$ is better than $c$ & $a$ is better than $e$ \\
\hline
\end{tabular}

Table 2: Comparison of site $a$ with sites $b, c$, and $d$ with respect to the image and access criteria

Given the information presented in Table 2, the DMR considers the weights $k_{4}$ and $k_{5}$ assigned to criteria $g_{4}$ and $g_{5}$ appropriate, when only one criterion, $g_{4}$ or $g_{5}$, supports a decision that one action is better than another one. However, he/she judges that the sum $k_{4}+k_{5}$ is too high to characterize the role of this criteria pair when both supports the decision that one action is better than another one, because in this case each criterion is weakened by the other due to the degree of redundancy between them. The comparisons provided by the DMR about actions $a, b, c$, and $e$ express his/her conviction that, if one action is better than another one with respect to criteria $g_{4}$ and $g_{5}$ conjointly, it would be interesting to be able to take this mutual weakening effect into account. As this reasoning shows, the classic concordance index is not able to take such a mutual weakening effect into account (for an illustrative example see Section 6). This effect can be taken into account by decreasing the weights $k_{4}$ and $k_{5}$ for the criteria $g_{4}$ and $g_{5}$, respectively, in the concordance index of the assertion " $a$ is at least as good as $e$ ", when both criteria intervene conjointly to make the assertion valid. In the following sections, the negative amount that must be added to $k_{4}+k_{5}$ to model this mutual weakening effect is denoted $k_{45}=k_{54}$.

\subsection{Launching a new digital camera model}

In this example, a manufacturer wants to introduce a new digital camera model on the market. As in the previous example, we assume that the DMR and the analyst decided to use an ELECTRE-type method. For this purpose a family of seven criteria $\left(g_{1}-g_{7}\right)$ is built:

$g_{1}$ : purchasing costs $($ cost $)[\mathrm{min}]$;

$g_{2}$ : weaknesses (fragility) $[\mathrm{min}]$;

$g_{3}$ : user friendliness of the controls (workability) [max];

$g_{4}$ : image quality (image) $[\max ]$

$g_{5}$ : aesthetics [max];

$g_{6}$ : volume [min];

$g_{7}$ : weight $[\mathrm{min}]$. 
As in the previous example and for the same reasons, indifference and preference thresholds, as well as weights, were associated to each one of the seven criteria. In discussion, the DMR and the analyst must again decide how the weights of the criteria cost $\left(g_{1}\right)$ and fragility $\left(g_{2}\right)$ should intervene in the comparison of the possible actions or camera models. A digital camera model $a$ can be compared to the remaining models $b, c$, and $d$, according to these two criteria $\left(g_{1}\right.$ and $\left.g_{2}\right)$, as is shown in Table 3.

\begin{tabular}{|l|c|c|c|}
\hline & $b$ & $c$ & $d$ \\
\hline$g_{1}$ & $a$ is better than $b$ & $a$ worse than $c$ & $a$ is better than $d$ \\
\hline$g_{2}$ & $a$ is better than $b$ & $a$ is better than $c$ & $a$ is worse than $d$ \\
\hline
\end{tabular}

Table 3: Comparison of model $a$ with models $b, c$ and $d$ with respect to cost and fragility

According to the classic definition of the concordance index (see Section 3), the role that criteria $g_{1}$ and $g_{2}$ should play in supporting the assertion "model $a$ is at least as good as model $b$ (or $c$ or $d$ )" is characterized by the following weights,

$k_{1}+k_{2}$ in the comparison with $b$,

$k_{2}$ in the comparison with $c$,

$k_{1}$ in the comparison with $d$.

The value of each of these weights was set without taking into account the impact that the other criterion's weight could have independent of whether or not it belongs to the concordant coalition; in other words, all the possible interactions between criteria are abstracted. Given the information in Table 3, the DMR considers that weights $k_{1}$ and $k_{2}$ adequately characterize the role these two criteria should play when comparing $a$ with $b$ and $a$ with $c$; however, he/she considers that the same is not true when comparing $a$ with $d$. Based on a customer survey, it seems that when one model is less fragile than another, the benefit derived from the lower cost is partially masked by the fact the model is less fragile. This phenomenon can be modeled by decreasing the weight of criterion $g_{1}$ in the concordance index of the assertion " $a$ is at least as good as $b$ ". In the following sections, the quantity that must be subtracted from $k_{1}$ to take into account this antagonistic effect (i.e., masking effect) of criterion $g_{2}$ with respect to criterion $g_{1}$ is denoted $k_{12}^{\prime}$.

Please note that if the DMR considers that the role of $g_{2}$ is adequately taken into account by the weight $k_{2}$ in the concordance index of the assertion " $a$ is at least as good as $c$ ", nothing can make him/her consider the possibility of an antagonistic effect of $g_{1}$ with respect to $g_{2}$. On the other hand, if the results of the customer survey justify taking such an antagonism into account, the quantity $k_{21}^{\prime}$ that must be subtracted from $k_{2}$ to model this interaction effect could be different from $k_{12}^{\prime}$. In other words, there is not necessarily symmetry between the two situations.

\section{Concepts: Definitions and notation}

This section presents some elementary concepts, definitions, and the notation used in the rest of this paper. As for the key concepts and the main features concerning ELECTRE methods (the context in which they are relevant, modeling with an outranking relation, their structure, the role of criteria, and how to account for imperfect knowledge) see Figueira et al. (2005). A comprehensive treatment of ELECTRE methods may be found in Roy and Bouyssou (1993) and Vincke (1992). Much of the theory developed on this field is presented in these books.

\subsection{Basic data}

The basic data of a multiple criteria problem is composed of a coherent set or family of criteria, a set of actions, and an evaluation matrix. Let,

- $F=\left\{g_{1}, g_{2}, \ldots, g_{i}, \ldots, g_{n}\right\}$ denote a family or set of criteria; for the sake of simplicity we shall use also $F$ as the set of criteria indices (the same will apply later on for subsets of $F$ );

- $A=\{a, b, c, \ldots\}$ denote a finite set of actions with cardinality $m$;

- $g_{i}(a) \in E_{i}$ denote the evaluation of action $a$ on criterion $g_{i}$, for all $a \in A$ and $i \in F$, where $E_{i}$ is the scale associated to criterion $g_{i}$ (no restriction is imposed to the scale type).

In what follows it is assumed that all the criteria are to be maximized, which is not a restrictive assumption. 


\subsection{Binary relations}

When comparing two actions $a$ and $b$, the following comprehensive binary relations can be defined on the set $A$. For a pair $(a, b) \in A \times A$ let,

- $P$ denote the strict preference relation; $a P b$ means that " $a$ is strictly preferred to $b$ ";

- I denote the indifference relation; $a I b$ means that " $a$ is indifferent to $b$ ";

- $Q$ denote the weak preference relation; $a Q b$ means that " $a$ is weakly preferred to $b$, which expresses hesitation between indifference $(I)$ and preference $(P)$;

- $S$ denote the outranking relation; $a S b$ means that " $a$ outranks $b$ " or more precisely that " $a$ is at least as good as $b$ ". Note that, $S=I \cup Q \cup P$.

For a given criterion $g_{i}$, the same interpretation of the above binary relations is valid, but now these relations are called partial binary relations, $P_{i}, I_{i}, Q_{i}$, and $S_{i}$, respectively.

\subsection{The notion of pseudo-criterion}

The concept of pseudo-criterion is based on the definition of two preference parameters, called thresholds. Let

- $q_{i}\left(g_{i}(a)\right)$ denotes the indifference threshold for criterion $g_{i}$, for all $a \in A$ and $i \in F$;

- $p_{i}\left(g_{i}(a)\right)$ denotes the preference threshold for criterion $g_{i}$, for all $a \in A$ and $i \in F$.

such that $p_{i}\left(g_{i}(a)\right) \geq q_{i}\left(g_{i}(a)\right)$, for all $g_{i}(a) \in E_{i}$ and $a \in A$.

Definition 1 (pseudo-criterion). A pseudo-criterion is a function $g_{i}$ associated with the two threshold functions $q_{i}\left(g_{i}(a)\right)$ and $p_{i}\left(g_{i}(a)\right)$ satisfying the following condition, for all $a \in A\left(\right.$ Roy, 1991, 1996): $g_{i}(a)+p_{i}\left(g_{i}(a)\right)$ and $g_{i}(a)+q_{i}\left(g_{i}(a)\right)$ are non-decreasing monotone function of $g_{i}(a)$.

By definition, for all pairs $(a, b) \in A \times A$ with $g_{i}(a) \geq g_{i}(b)$,

$$
\begin{aligned}
a I_{i} b & \Leftrightarrow g_{i}(a) \leq g_{i}(b)+q_{i}\left(g_{i}(b)\right) \\
a Q_{i} b & \Leftrightarrow g_{i}(b)+q_{i}\left(g_{i}(b)\right)<g_{i}(a) \leq g_{i}(b)+p_{i}\left(g_{i}(b)\right) \\
a P_{i} b & \Leftrightarrow g_{i}(b)+p_{i}\left(g_{i}(b)\right)<g_{i}(a) .
\end{aligned}
$$

Definition 2 (quasi criterion). If, $q_{i}\left(g_{i}(a)\right)=p_{i}\left(g_{i}(a)\right)$, for all $a \in A$, then $g_{i}$ is called a quasi criterion. It is a particular case of a pseudo criterion which is also considered in the rest of the paper. For a quasi criterion there is no ambiguity zone, that is, there is no weak preference $Q_{i}$.

In what follows $C(a T b)$ represents the coalition of criteria in favor of the assertion " $a T b$ ", where $T \in\{P, Q, S\}$

\subsection{The criteria weights and the concordance index}

In ELECTRE methods, the relative importance coefficients attached to the criteria refer to intrinsic weights. For a given criterion $g_{i}$, the weight $k_{i}, k_{i}>0$ for all $g_{i} \in F$, can be interpreted as its voting power when it contributes to the majority which is in favor of an outranking; it is not a substitution rate. For more details about the question on how to attribute numerical values to the parameters which must reflect the relative importance of criteria, see Figueira and Roy (2002), Mousseau (1993, 1995) and Roy and Mousseau (1996).

ELECTRE Multiple Criteria Aggregation Procedures (MCAPs) are based on a concordance index $c(a, b)$ which is used both to validate the assertion " $a$ outranks $b$ " and/or to give a measure of the credibility of such an assertion. The concordance index can be defined as follows, 


$$
c(a, b)=\sum_{i \in C(a S b)} \frac{k_{i}}{K}, \text { where } K=\sum_{i \in F} k_{i}
$$

and, $C(a S b)$ represents the coalition of criteria in favor of the assertion " $a$ outranks $b$ ", when $F$ if composed of quasi criteria.

When $F$ contains at least a pseudo-criterion, this index should be rewritten in the following way (as in ELECTRE IS, III, and TRI, see Figueira et al., 2005),

$$
c(a, b)=\sum_{i \in F} \frac{k_{i}}{K} c_{i}(a, b)
$$

where,

$$
c_{i}(a, b)=\left\{\begin{array}{ccc}
1, & \text { if } \quad g_{i}(a)+q_{i}\left(g_{i}(a)\right) \geq g_{i}(b), \quad\left(a S_{i} b\right), \\
\frac{g_{i}(a)+p_{i}\left(g_{i}(a)\right)-g_{i}(b)}{p_{i}\left(g_{i}(a)\right)-q_{i}\left(g_{i}(a)\right)}, & \text { if } \quad & g_{i}(a)+q_{i}\left(g_{i}(a)\right)<g_{i}(b) \leq g_{i}(a)+p_{i}\left(g_{i}(a)\right), \quad\left(b Q_{i} a\right), \\
0, & \text { if } \quad & g_{i}(a)+p_{i}\left(g_{i}(a)\right)<g_{i}(b), \quad\left(b P_{i} a\right) .
\end{array}\right.
$$

It is easy to see that, when $F$ is composed of quasi criteria, index (2) becomes (1).

Let $\bar{C}(b P a)$ denote the complement of $C(b P a)$. It should be remarked that when $F$ comprises only quasi criteria $\bar{C}(b P a)=C(a S b)$; if $F$ is composed of at least one pseudo-criterion $\bar{C}(b P a)=C(a S b) \cup C(b Q a)$. In both cases this set represents the coalition of all the criteria which are not strongly opposed to the assertion $a S b$ (let us recall that $b Q a$ is not a strong opposition).

\subsection{Properties of $c(a, b)$}

The following properties of $c(a, b)$ hold for all pairs $(a, b) \in A \times A$,

Boundary conditions: $0 \leq c(a, b) \leq 1$.

Monotonicity: $c(a, b)$ is a monotonous non-decreasing function of $\Delta_{i}=g_{i}(a)-g_{i}(b)$, for all $i \in F$.

Continuity: if $p_{i}\left(g_{i}(a)\right)>q_{i}\left(g_{i}(a)\right)$, for all $i \in F$ and $a \in A$, then $c(a, b)$ is a continuous function of both $g_{i}(a)$ and $g_{i}(b)$.

The proof of the boundary conditions is obvious. The proof of monotonicity is based on the fact that, for each $i$, $c_{i}(a, b)$ has the same property. Continuity is not valid for quasi criteria. The proof for the case of pseudo-criteria is also based on the fact that, for each $i, c_{i}(a, b)$ has the same property.

\section{Types of interactions considered}

The above formulae (1) and (2) do not take any type of dependency between the considered criteria into account. Very often, this is justified because the formulae are used to deal with a structural dependence related to various points concerning distinct stakeholder (Roy and Bouyssou, 1993). For the sake of the clarity, a coherent criteria family must be defined so as to reduce other types of dependency as much as possible (see, for example, Bisdorff, 2001). It is also necessary to completely remove any dependencies derived from dispersion or from a classical utility approach (Roy and Bouyssou, 1993). Consequently, from a practical point of view, the dependencies that really need to be taken into account are not numerous and in general concern only criteria pairs. Considering criteria triples or quadruples and so on would be 
too complicated to be effective in a decision aiding process because formulating them would involve so many problems of interpretation and comprehension that their expected added value would vanish (see Roy, 2007).

Therefore, we consider the cases where the only dependencies between criteria which deserve to be taken into account in MCAPs are related to interactions between criteria pairs. In this paper we are interested in the situations in which the interactions can be modeled using one of the three interaction types presented below. These definitions are modifications of formulae (1) and (2). The conditions in which these modifications take place are related to a given interaction type. This work is based on the research of Greco and Figueira (2003), in which similar interaction types can be found. Roy (2007) provides a more general formulation of the three types of interaction proposed in this paper, which is independent of the ways the interactions are taken into account in the concordance index.

\subsection{Definitions}

This section provides the definitions of the three interaction types. Let us notice that case $a-b$ is mutually exclusive, but cases $a-c$ and $b-c$ are not.

a) Mutual strengthening effect

If criteria $g_{i}$ and $g_{j}$ both strongly, or even weakly, support the assertion $a S b$ (more precisely, $g_{i}, g_{j} \in \bar{C}(b P a)$ ), we consider that their contribution to the concordance index must be larger than the sum of $k_{i}+k_{j}$, because these two weights represent the contribution of each of the two criteria to the concordance index when the other criterion does not support $a S b$. We suppose that the effect of the combined presence of $g_{i}$ and $g_{j}$ among the criteria supporting the assertion $a S b$ can be modeled by a mutual strengthening coefficient $k_{i j}>0$, which intervenes algebraically in $c(a, b)$. (For an example, see the interaction between $g_{1}$ and $g_{2}$ in Section 2.1.) Please note that $k_{i j}=k_{j i}$.

\section{b) Mutual weakening effect}

If criteria $g_{i}$ and $g_{j}$ both strongly, or even weakly, support the assertion $a S b$ (more precisely, $g_{i}, g_{j} \in \bar{C}(b P a)$ ), we consider that their contribution to the concordance index must be smaller than the sum of $k_{i}+k_{j}$, because these two weights represent the contribution of each of the two criteria to the concordance index when the other criterion does not support $a S b$. We suppose that this effect can be modeled using a mutual weakening coefficient $k_{i j}<0$, which intervenes algebraically in $c(a, b)$. (For an example, see the interaction between $g_{3}$ and $g_{4}$ in Section 2.1.) Please note that $k_{i j}=k_{j i}$.

\section{c) Antagonistic effect}

If criterion $g_{i}$ strongly, or weakly, supports the assertion $a S b$ and criterion $g_{h}$ strongly opposes this assertion, we consider that the contribution of the criterion $g_{i}$ to the concordance index must be smaller than the weight $k_{i}$ that was considered in cases in which $g_{h}$ does not belong to $C(b P a)$. We suppose that this effect can be modeled by introducing an antagonism coefficient $k_{i h}^{\prime}>0$, which intervenes negatively in $c(a, b)$. (For an example, see the "cost" and "fragility" criteria in Section 2.2., where "cost" is $g_{i}$ and "fragility" is $g_{h}$.) Please note that the presence of an antagonism coefficient $k_{i h}^{\prime}>0$ is compatible with both the absence of antagonism in the reverse direction $\left(k_{h i}^{\prime}=0\right)$ and the presence of a reverse antagonism $\left(k_{h i}^{\prime}>0\right)$.

The antagonistic effect does not double the influence of the veto effect; in fact, they are quite different. If criterion $g_{h}$ has a veto power, it will always be considered, regardless of whether $g_{i}$ belongs to the concordant coalition. The same is not true for the antagonistic effect, which occurs only when the criterion $g_{i}$ belongs to the concordant coalition. Let us notice the a veto effect or threshold expresses the power attributed to a given criterion, $g_{j}$ to be against the assertion "a outranks b", when the difference of the evaluation between $g_{j}(b)$ and $g_{j}(a)$ is greater than this threshold.

\subsection{Practical aspects}

The four-step procedure presented in this section shows how numerical values can be assigned to the parameters introduced below in order to characterize the mutual strengthening, mutual weakening, and the antagonistic effects. The parameters were designed so that these effects could be taken into account in the ELECTRE methods that use of the concordance index, as mentioned in Section 3. The four-step procedure is used in the context of a constructive perspective when using ELECTRE methods rather than a descriptive one (cf. Roy, 1993, 2005, 2007). 
Step 1 As is traditional in the ELECTRE method, step 1 assigns numerical values to the intrinsic weights $k_{i}, i=1, \ldots, n$. The revised "pack of cards" method can be used for such a purpose (see SRF software by Figueira and Roy, 2002). The analyst should, however, point out to the DMR that the value of the relative weight $k_{i}$ should be set without taking into account the impact that certain criteria, regardless of whether they belong to the concordant coalition, could have. In other words, the "cards" should be ranked ignoring all the possible kinds of inter-criteria interaction (cf. Section 2).

Step 2 The analyst should ask the DMR about the possible interactions that he/she thinks must be taken into account. In order to make sure that the DMR has a good understanding of the interaction effects, the analyst can use illustrative examples like the ones presented in Section 1. Then, considering criterion $g_{1}$ and reviewing the remaining criteria $g_{2}, g_{3}, \ldots, g_{n}$, it should be easy (and relatively quick), given the very nature of the criteria, to recognize:

- That considering an interaction between $g_{1}$ and another criterion is not justified, or

- That an interaction between $g_{1}$ and at least one of the remaining criteria must be considered. In this case, it is also necessary to define which kind of interaction exists: mutual strengthening, mutual weakening, or antagonistic. For our purposes here, we assume that antagonistic interaction excludes the presence of the other two types, in which case, the sign of the interaction(s) must also be defined (cf. $4.1 \mathrm{c}$ ).

This procedure is repeated to consider the possible interactions between $g_{2}$ and $g_{3}, \ldots, g_{n}$, then between $g_{3}$ and $g_{4}, \ldots, g_{n}$, and, finally between $g_{n-1}$ and $g_{n}$.

If the criteria family is appropriately designed, the number of pairs/ordered pairs for which an interaction effect can be defined should be rather very small.

Step 3 A numerical value is assigned to the interaction coefficient associated with each pair identified in the previous step. As stated in the coefficients' definition (cf. Sections 2 and 4.1), the larger their absolute value, the more important the interaction effect. By definition, these coefficients are defined, such that:

If there is a mutual strengthening or a mutual weakening effect between criteria $g_{i}$ and $g_{j}$, then the relative weights of these two criteria in $c(a, b)$ should be $k_{i}+k_{j}+k_{i j}$ instead of $k_{i}+k_{j}\left(c f\right.$. Section 3) as soon as $a S_{i} b$ and $a S_{j} b$ is found.

If criterion $g_{h}$ has an antagonistic effect with respect to criterion $g_{i}$, then the relative weight of criterion $g_{i}$ in $c(a, b)$ should be $k_{i}-k_{i h}^{\prime}$ instead of $k_{i}$ (cf. Section 3) as soon as $a S_{i} b$ and $b P_{h}$ a is found.

These definitions should be considered to support the analyst's position on the appropriate value of each interaction coefficient to take the importance of the effect the DMR considers appropriate (cf. Step 2) into account in the model. Let us review the examples presented in Section 2 to show how the analyst should proceed.

Case 1 (mutual strengthening effect): cf. $2.1 a$, criteria $g_{1}$ and $g_{2}$. Suppose that when using SRF the result is $k_{1}=5$ and $k_{2}=4$, and thus $k_{1}+k_{2}=9$. Since there is a mutual strengthening effect, the relative weights of these two criteria should be larger than 9, when comparing the two sites $a$ and $d$ (cf. Table 1). The analyst can ask the DMR to set the value to be replaced to 9 in this comparison in order to adequately model the interaction that the DMR wants to take into account. If the answer is 12 , for example, the analyst should conclude that $k_{12}=3$.

Case 2 (mutual weakening effect): cf. $2.1 \mathrm{~b}$, criteria $g_{4}$ and $g_{5}$. Suppose that when using SRF the result is $k_{4}=3$ and $k_{5}=3$, and thus $k_{4}+k_{5}=6$. Since there is a mutual weakening effect, the relative weights of these two criteria should be lower than 6, when comparing the two sites $a$ and $e$ (cf. Table 2). The analyst can ask the DMR to set the value to be replaced to 6 in this comparison in order to adequately model the interaction that the DMR wants to take into account. If the answer is 4 , for example, the analyst should conclude that $k_{45}=-2$.

Case 3 (antagonistic effect): cf. 2.2, criteria $g_{1}$ and $g_{2}$. Suppose that when using SRF the result is $k_{1}=6$ and $k_{2}=4$, and thus $k_{1}+k_{2}=10$. Since criterion $g_{2}$ is antagonistic with respect to $g_{1}$, the weight $k_{1}$ should be lower than 6 , when comparing two digital camera models $a$ and $d$ (cf. Table 3). The analyst can ask the DMR to set the 
value to be replaced to 6 in this comparison in order to adequately model the interaction that the DMR wants to take into account. If the answer is 3.5 , for example, the analyst should conclude that $k_{12}^{\prime}=2.5$.

Please note that the procedure followed for the latter case is a little bit different from the other two cases, which only underlines the difference between the antagonistic effect and the mutual strengthening and mutual weakening effects. This difference is connected to the fact that, when $k_{i h}^{\prime} \neq 0$, it is also possible to have $k_{h i}^{\prime}=0$ or $k_{h i}^{\prime} \neq 0$ (cf. $4.1 \mathrm{c})$, without requiring that $k_{i h}^{\prime}=k_{h i}^{\prime}$.

The antagonistic effect that may exist between two criteria $g_{i}$ and $g_{h}$ can be formally taken into account as a mutual strengthening effect between these two criteria. In this case, the initial weights $k_{i}$ and $k_{h}$, obtained using SRF, should be replaced with the values $k_{i}-k_{i h}^{\prime}$ and $k_{h}-k_{h i}^{\prime}$ respectively, such that $k_{i h}=k_{i h}^{\prime}+k_{h i}^{\prime}$. However, this ploy, which is very difficult for those who use it, does not prevent the antagonistic effect from being used to define the values $k_{i h}^{\prime}$ and $k_{h i}^{\prime}$. In addition, as will be shown at the end of Section 5, this equivalence is not valid for pseudo-criteria.

Step 4 In this step the net balance condition is checked, because, in very specific cases, an improper result can mean a return to the previous step to modify the value assigned to some interaction coefficients.

Let $k_{i j}$ be the negative value of the interaction coefficient used to characterize a mutual weakening effect. Since the interaction can, at most, render the contribution of criterion $g_{i}$ to $c(a, b)$ null when $a S_{i} b$ and $a S_{j} b$, the following should be true:

$$
k_{i}-\left|k_{i j}\right| \geq 0
$$

In the same way, since this interaction can, at most, render the contribution of criterion $g_{i}$ to $c(a, b)$ null when $a S_{i} b$ and $b P_{h} a$, the interaction coefficient $k_{i h}^{\prime}$ that allows an antagonistic effect to be characterized should be defined such that:

$$
k_{i}-k_{i h}^{\prime} \geq 0
$$

Suppose the two previous interactions, where criterion $g_{i}$ is present, were considered. When $a S_{i} b, a S_{j} b$, and $b P_{h} a$ simultaneously occurs, the contribution of the three criteria to $c(a, b)$ is equal to:

$$
k_{i}-k_{i h}^{\prime}-\left|k_{i j}\right|
$$

This quantity must be positive since the two interactions taken into account cannot render the contribution of $g_{i}$ to $c(a, b)$ non-negative. Thus, in Step 3, the analyst should check whether or not the values assigned to the interaction coefficients fulfill the previous inequalities. The different types of interaction considered here with $g_{i}$ can be present not only with one criterion $g_{j}$ or $g_{h}$, but with two or even, exceptionally, with three or more. Thus the analyst should check that, for each criterion $g_{i}$ that interacts with several criteria, the following net balance condition is fulfilled:

Condition (positive net balance). For all $i \in F$,

$$
\left(k_{i}\right)-\left(\sum_{\{i, j\}: k_{i j}<0}\left|k_{i j}\right|+\sum_{h} k_{i h}^{\prime}\right)>0
$$

If a criterion $g_{i}$ for which this inequality is not fulfilled, the values of the interaction coefficients shown in brackets should be questioned. Clearly, the number of pairs of interaction criteria is generally small, thus the inequalities that must be verified, if any exist, are also quite few. 


\section{Extensions of the concordance index}

This section is devoted to the definition of the concordance index, first when $F$ is composed of quasi criteria, and then when at least one criterion is a pseudo-criterion.

Before presenting the formulae it is useful to introduce the following additional notation. Let,

- $L(a, b)$ denote the set of all pairs $\{i, j\}$ such that $i, j \in \bar{C}(b P a)$;

- $O(a, b)$ denote the set of all ordered pairs $(i, h)$ such that $i \in \bar{C}(b P a)$ and $h \in C(b P a)$.

\subsection{The quasi criterion model}

Let us recall that a quasi criterion is a pseudo-criterion such that $q_{i}\left(g_{i}(a)\right)=p_{i}\left(g_{i}(a)\right)$, for all $a \in A$.

\subsubsection{Definition of $c(a, b)$}

The comprehensive concordance index, when $F$ is composed of quasi criteria, is defined as follows,

$$
c(a, b)=\frac{1}{K(a, b)}\left(\sum_{i \in \bar{C}(b P a)} k_{i}+\sum_{\{i, j\} \in L(a, b)} k_{i j}-\sum_{(i, h) \in O(a, b)} k_{i h}^{\prime}\right)
$$

where,

$$
K(a, b)=\sum_{i \in F} k_{i}+\sum_{\{i, j\} \in L(a, b)} k_{i j}-\sum_{(i, h) \in O(a, b)} k_{i h}^{\prime}
$$

Note that, in general, $K(a, b) \neq K(b, a)$. Observe also that, from positive balance condition $K(a, b)>0$ is always true.

As for the new definition of $c(a, b)$, the following properties should be fulfilled.

Coherence: The definition of $c(a, b)$ as in formula (4) should be coherent with the classical definition of the $c(a, b)$ as it was presented in Section 3.

It means that, when we compare two actions $a$ and $b$ and when there is no interaction effect regarding this comparison, the new $c(a, b)$ should be the same as the one of Section 3. The proof is quite obvious since when there is no interaction effect, $L(a, b)=\emptyset$ and $O(a, b)=\emptyset$, and consequently $c(a, b)$ in formula (4) becomes, $c(a, b)=\frac{1}{K(a, b)} \sum_{i \in F} k_{i}$ with $K(a, b)=K$.

Boundary conditions: $0 \leq c(a, b) \leq 1$.

As for the proof let us consider separately the two inequalities,

1. $c(a, b) \geq 0$

This inequality derives from the definition of $c(a, b)$ and the net balance condition; it is fulfilled for every $K(a, b)$. The proof is provided in Theorem 1 .

2. $c(a, b) \leq 1$

Two cases have to be considered,

(a) $\bar{C}(b P a)=F$ (all the criteria belong to the concordant coalition)

It represents unanimity and the index must be equal to one,

$$
c(a, b)=1
$$

Since unanimity leads to the absence of antagonistic interaction effects, $c(a, b)$ can be rewritten as follows,

$$
c(a, b)=\frac{1}{K(a, b)}\left(\sum_{i \in F} k_{i}+\sum_{\{i, j\} \in L(a, b)} k_{i j}\right)=1
$$


(b) $\bar{C}(b P a) \neq F$ (at least one criterion belongs to $C(b P a)$ )

In the previous case, the antagonistic coefficients were not present. As soon as these coefficients appear in $c(a, b)$ it becomes strictly lower than 1, i.e., $c(a, b)<1$.

Remark 4. If $F$ is composed of quasi criteria, the function $c(a, b)$ presents a discontinuity when $g_{i}(a)+q_{i}\left(g_{i}(a)\right)$ becomes strictly lower than $g_{i}(b)$. In the case of pseudo-criteria, $p_{i}\left(g_{i}(a)\right)>q_{i}\left(g_{i}(a)\right)$, for all $i \in F$ and $a \in A$, this discontinuity will not occur.

\subsubsection{Main theorem}

Let us consider the pair $(a, b) \in A \times A$ and calculate the following algebraic sum,

$$
S(a, b)=\sum_{i \in \bar{C}(b P a)} k_{i}+\sum_{\{i, j\} \in L(a, b)} k_{i j}-\sum_{(i, h) \in O(a, b)} k_{i h}^{\prime}
$$

Lemma 1. For all $(a, b) \in A \times A$ and for all $f \in F, S(a, b)$ is a monotone non-decreasing function of $\Delta_{f}$ and $S(a, b) \geq 0$. (The proof of this lemma is provided in the Appendix)

Remembering that $\bar{C}(b P a)$ can be any subset $E \subseteq F$, the non-negativity of $S(a, b)$ proved in Lemma 1 can be rewritten as follows, for all $E \subseteq F$,

$$
\sum_{i \in E} k_{i}+\sum_{\{i, j\} \in E} k_{i j}-\sum_{i \in E, h \in F \backslash E} k_{i h}^{\prime} \geq 0
$$

Before introducing the main result it is important to establish also the following lemma.

Lemma 2. For all $(a, b) \in A \times A$ and for all $f \in F, c(a, b)$ defined as in (4) is a monotone non-decreasing function of $\Delta_{f}$ if and only if the non-negativity summation condition is fulfilled. (The proof of this lemma is provided in the Appendix)

The main result is established in the following theorem.

Theorem 1. Monotonicity and boundary conditions hold for $c(a, b)$ as defined in formula (4).

Proof.

Lemma 2 proves monotonicity. Let us now prove the boundary conditions,

1. $c(a, b) \geq 0$

If $\bar{C}(b P a)=\varnothing$, then $c(a, b)=0$. Suppose that we could have $c(a, b)<0$. This implies that at least one criterion $f$ does not belong to $\bar{C}(b P a) \neq \varnothing$. Consider that there exists at least one criterion in $\bar{C}(b P a)$. If for all $f$ in $\bar{C}(b P a), \Delta_{f}$ is forced to decrease till $\bar{C}(b P a)=\varnothing$, then $c(a, b)$ cannot increase. Contradiction!

2. $c(a, b) \leq 1$

From condition (5), $c(a, b)=1$, when $\bar{C}(b P a)=F$. Suppose that we could have $c(a, b)>1$. This implies that at least one criterion $f$ does not belong to $\bar{C}(b P a)$. Consider that there exists at least one criterion that does not belong to $\bar{C}(b P a)$. If for all $f, \Delta_{f}$ is forced to increase till $f$ becomes an element of $\bar{C}(b P a)$, then $c(a, b)$ cannot decrease. Contradiction!

The proof is now complete.

\subsection{The pseudo-criterion model}

When dealing with a pseudo-criterion, $g_{i}$, an ambiguity zone should be taken into account, for all $(a, b) \in A \times A$,

$$
g_{i}(a)+q_{i}\left(g_{i}(a)\right)<g_{i}(b) \leq g_{i}(a)+p_{i}\left(g_{i}(a)\right)
$$




\subsubsection{Definition of $c(a, b)$}

The definition of $c(a, b)$ can be stated in the following manner,

$$
c(a, b)=\frac{1}{K(a, b)}\left(\sum_{i \in \bar{C}(b P a)} c_{i}(a, b) k_{i}+\sum_{\{i, j\} \in L(a, b)} Z\left(c_{i}(a, b), c_{j}(a, b)\right) k_{i j}-\sum_{(i, h) \in O(a, b)} Z\left(c_{i}(a, b), c_{h}(b, a)\right) k_{i h}^{\prime}\right)
$$

where,

$$
K(a, b)=\sum_{i \in F} k_{i}+\sum_{\{i, j\} \in L(a, b)} Z\left(c_{i}(a, b), c_{j}(a, b)\right) k_{i j}-\sum_{(i, h) \in O(a, b)} Z\left(c_{i}(a, b), c_{h}(b, a)\right) k_{i h}^{\prime}
$$

Function $Z(\cdot, \cdot)$ in formula (6) is used to capture the interaction effects in the ambiguity zone. It should be remarked that in the third summation $c_{h}(b, a)$ is always equal to 1 .

Remark 5. For the sake of clarity and simplicity, the same function $Z(\cdot, \cdot)$ is used in both, the second and the third summations. It would, however, be possible to use different functions.

Let $x=c_{i}(a, b)$ and $y=c_{j}(a, b)$ or $y=c_{h}(b, a)$. Consequently, $x, y \in[0,1]$. Function $Z(x, y)$ is used to get the reduction coefficients for $k_{i j}$ and $k_{i h}^{\prime}$ when, at least one of the arguments of $Z(x, y)$ is within the range ]0,1[.

What are the properties of $Z(x, y)$ to guarantee the coherence of formula (6)?

Extreme value conditions: When leaving the ambiguity zones, $c(a, b)$ should regain the form presented in formula (4). Thus, $Z(1,1)=1$ and $Z(x, 0)=Z(0, y)=0$.

Symmetry: For the same reason for put $k_{i j}=k_{j i}$, since we assume that also $Z(x, y)=Z(y, x)$.

Monotonicity: When the ambiguity diminishes, the effect due to the interaction cannot increase. Then $Z(x, y)$ is a non-decreasing monotone function of both arguments $x$ and $y$.

Marginal impact condition: When the ambiguity diminishes passing from $x+w$ to $x$, the relative marginal impact of the interactions is bounded from above,

$$
\frac{1}{w}(Z(x+w, y)-Z(x, y)) \leq 1 \quad x, y, w, x+w \in[0,1]
$$

We will see the interest of this condition in the proof of Lemma 3 (see Appendix).

Continuity: Formula (2) is a continuous function of $g_{i}(a)$ and $g_{i}(b)$ when $p_{i}\left(g_{i}(a)\right)>q_{i}\left(g_{i}(a)\right)$, for all $a \in A$. If we want to preserve continuity, then it is necessary that $Z(x, y)$ is a continuous function of each argument.

Boundary condition: For preserving the net balance condition, it is sufficient that $Z(x, y) \leq \min \{x, y\}$.

The boundary condition is a particular case of the marginal impact condition. The proof is as follows.

$$
\frac{1}{w}(Z(x+w, y)-Z(x, y)) \leq 1 \Leftrightarrow Z(x+w, y)-Z(x, y) \leq w
$$

Consider now, $x=0$

$$
Z(0+w, y)-Z(0, y) \leq w
$$

and, according to the extreme value conditions,

$$
Z(w, y)-0 \leq w \Leftrightarrow Z(w, y) \leq w
$$

Now, for symmetry, we get

$$
Z(w, y) \leq \min \{w, y\}
$$


Therefore, it is sufficient to consider only the marginal impact condition. However, for the sake of a better comprehension, we keep both conditions; it sounds more intuitive.

Among the multiple forms that can be chosen for $Z(x, y)$, we only present two of them which have an intuitive and meaningful interpretation.

$$
\begin{aligned}
& Z(x, y)=\min \{x, y\} \\
& Z(x, y)=x y .
\end{aligned}
$$

If $x$ and/or $y$ are equal to 1, both formulae are equivalent. But, when $x$ and $y$ are both different from 1, that is, when the two interacting criteria belong to the ambiguity zone, then the impact of the interaction is weaker with $x y$ than with $\min \{x, y\}$. Choosing the $\min \{x, y\}$ formula means that the reduction coefficient is not influenced by what happens in the other ambiguity zone. For these reasons the formula $x y$ seems preferable to $\min \{x, y\}$.

\subsubsection{Extension of the main theorem}

This section presents an extension of the previous results when $F$ is composed of at least one pseudo-criterion. The proofs are similar to the ones provided for Lemma 2 and Theorem 1.

Let us consider the pair $(a, b) \in A \times A$ and calculate the following algebraic sum,

$$
S(a, b)=\sum_{i \in \bar{C}(b P a)} c_{i}(a, b) k_{i}+\sum_{\{i, j\} \in L(a, b)} Z\left(c_{i}(a, b), c_{j}(a, b)\right) k_{i j}-\sum_{(i, h) \in O(a, b)} Z\left(c_{i}(a, b), c_{h}(b, a)\right) k_{i h}^{\prime}
$$

Lemma 3. For all $(a, b) \in A \times A$ and for all $f \in F, S(a, b)$ is a monotone non-decreasing function of $\Delta_{f}$ and $S(a, b) \geq 0$. (The proof of this lemma is provided in the Appendix)

An immediate consequence of Lemma 3 is that $K(a, b)>0$ for all $(a, b) \in A \times A$. In fact

$$
K(a, b)=S(a, b)+\left(\sum_{i \in \bar{C}(b P a)}\left(1-c_{i}(a, b)\right) k_{i}+\sum_{j \in C(b P a)} k_{j}\right)
$$

Two cases are possible $(\varnothing \subset C(a S b) \subseteq F)$ :

- $C(a S b) \subseteq F$ : in this case the quantity in between big parentheses is always positive because $k_{i}>0$ for all $g_{i} \in F$, such that the non-negativity of $S(a, b)$ implies that $K(a, b)>0$;

- $C(a S b)=\varnothing$ : in this case the $K(a, b)=\sum_{i \in F} k_{i}>0$ because $k_{i}>0$, for all $g_{i} \in F$.

Lemma 4. For all $(a, b) \in A \times A$ and for all $f \in F, c(a, b)$ defined as in (6) is a monotone non-decreasing function of $\Delta_{f}$. (The proof of this lemma is provided in the Appendix)

Now the main result can be established.

Theorem 2. Boundary conditions, monotonicity, and continuity hold for $c(a, b)$ as defined in formula (6).

Proof.

Lemma 2 establishes monotonicity. Boundary conditions hold when considering pseudo-criteria. And continuity derives from the fact that,

1. the functions $c_{f}(a, b), Z(x, y)$ are continuous, and

2. the conditions $c_{f}(a, b)=0$ if $g_{f}(a)+q_{f}\left(g_{f}(a)\right)-g_{f}\left(g_{f}(b)\right)=0$ and $Z(0, y)=Z(x, 0)=0$ guarantees continuity when a criterion becomes a member or when it is removed from one of the following sets, $\bar{C}(a, b), L(a, b)$, or $O(a, b)$.

The proof is thus complete for the general case.

We complete this section (cf. end of Step 3 in Section 4.2) by showing that when dealing with pseudo-criteria the antagonistic effect is not equivalent to mutual strengthening. For the sake of simplicity, consider a decision problem with only two criteria, $g_{i}$ and $g_{h}$, and the following three cases: 
a) $g_{i}, g_{h} \in \bar{C}(b P a)$

b) $g_{i} \in \bar{C}(b P a)$ and $g_{h} \in C(b P a)$;

c) $g_{h} \in \bar{C}(b P a)$ and $g_{i} \in C(b P a)$.

Let us consider modeling cases $a), b)$, and $c$ ) in terms of both mutual strengthening, using the weights $k_{i}, k_{k}$, and $k_{i h}$, and antagonism, using the weights $\bar{k}_{i}, \bar{k}_{h}, \bar{k}_{i h}^{\prime}$, and $\bar{k}_{h i}^{\prime}$.

Taking into account modeling in terms of mutual strengthening and considering the concordance index $c(a, b)$, we have (following the above three cases):

a) $c(a, b)=\frac{k_{i} c_{i}(a, b)+k_{h} c_{h}(a, b)+k_{i h} Z\left(c_{i}(a, b), c_{h}(a, b)\right)}{k_{i}+k_{h}+k_{i h} Z\left(c_{i}(a, b), c_{h}(a, b)\right)}$

b) $c(a, b)=\frac{k_{i} c_{i}(a, b)}{k_{i}+k_{h}}$

c) $c(a, b)=\frac{k_{h} c_{h}(a, b)}{k_{i}+k_{h}}$.

Taking into account modeling in terms of antagonism and considering the concordance index $c(a, b)$, we have (following the above three cases):
a) $c(a, b)=\frac{\bar{k}_{i} c_{i}(a, b)+\bar{k}_{h} c_{h}(a, b)}{\bar{k}_{i}+\bar{k}_{h}}$
b) $c(a, b)=\frac{\bar{k}_{i} c_{i}(a, b)-\bar{k}_{i h}^{\prime} Z\left(c_{i}(a, b), c_{h}(b, a)\right)}{\bar{k}_{i}+\bar{k}_{h}-\bar{k}_{i h}^{\prime} Z\left(c_{i}(a, b), c_{h}(b, a)\right)}$;
c) $c(a, b)=\frac{\bar{k}_{h} c_{h}(a, b)-\bar{k}_{h i}^{\prime} Z\left(c_{h}(a, b), c_{i}(b, a)\right)}{\bar{k}_{i}+\bar{k}_{h}-\bar{k}_{h i}^{\prime} Z\left(c_{h}(a, b), c_{i}(b, a)\right)}$.

To get an equivalence between modeling in terms of mutual strengthening and antagonism, the following equations should hold for all the values of $c_{i}(a, b)$ and $c_{h}(a, b)$ in the above cases, i.e.,

a) $\frac{k_{i} c_{i}(a, b)+k_{h} c_{h}(a, b)+k_{i h} Z\left(c_{i}(a, b), c_{h}(a, b)\right)}{k_{i}+k_{h}+k_{i h} Z\left(c_{i}(a, b), c_{h}(a, b)\right)}=\frac{\bar{k}_{i} c_{i}(a, b)+\bar{k}_{h} c_{h}(a, b)}{\bar{k}_{i}+\bar{k}_{h}}$

b) $\frac{k_{i} c_{i}(a, b)}{k_{i}+k_{h}}=\frac{\bar{k}_{i} c_{i}(a, b)-\bar{k}_{i h}^{\prime} Z\left(c_{i}(a, b), c_{h}(b, a)\right)}{\bar{k}_{i}+\bar{k}_{h}-\bar{k}_{i h}^{\prime} Z\left(c_{i}(a, b), c_{h}(b, a)\right)}$;

c) $\frac{k_{h} c_{h}(a, b)}{k_{i}+k_{h}}=\frac{\bar{k}_{h} c_{h}(a, b)-\bar{k}_{h i}^{\prime} Z\left(c_{h}(a, b), c_{i}(b, a)\right)}{\bar{k}_{i}+\bar{k}_{h}-\bar{k}_{h i}^{\prime} Z\left(c_{h}(a, b), c_{i}(b, a)\right)}$.

Notice that the values of the weights $k_{i}, k_{h}, k_{i h}$ and $\bar{k}_{h}, \bar{k}_{i h}^{\prime}, \bar{k}_{h i}^{\prime}$, ensuring that the above equations hold, depend on the values of $c_{i}(a, b)$ and $c_{h}(a, b)$. This means that there are no weights $k_{i}, k_{h}, k_{i h}$ and $\bar{k}_{h}, \bar{k}_{i h}^{\prime}, \bar{k}_{h i}^{\prime}$ giving the same values of $c(a, b)$ when modeling in terms of mutual strengthening and when modeling in terms of antagonism, for all the possible values of $c_{i}(a, b)$ and $c_{h}(a, b)$. Therefore, in presence of pseudo-criteria, mutual strengthening, and antagonism are not equivalent.

\section{Modeling the interaction effects in the illustrative examples}

In this section, the impact on the pairwise comparisons of the three interactions effects illustrated in Section 2 is shown. When taking such effects into account, the comparisons between actions can change. In the following sub-sections, it is assumed that there is no veto effect in the pairwise comparisons of the actions. 


\subsection{Choosing a site for a new hotel construction project}

Table 4 presents the evaluations of the five sites $-a, b, c, d$, and $e$-according to the five criteria. In this example,

- The evaluations of criterion $g_{1}$ (investment costs) are expressed in thousands of $€$, designated $\mathrm{K} €$. The indifference and the preference thresholds assigned to this criterion are $q_{1}\left(g_{1}(x)\right)=500+0.03 g_{1}(x) \mathrm{K} €$ and $p_{1}\left(g_{1}(x)\right)=$ $1000+0.05 g_{1}(x) \mathrm{K} €$, respectively, where $x$ is the worst of the two actions (c.f. Section 3.3).

- The evaluations of criterion $g_{2}$ (annual costs) are also expressed in $\mathrm{K} €$; the thresholds assigned to this criterion are $q_{2}\left(g_{1}(x)\right)=50+0.05 g_{1}(x) \mathrm{K} €$ and $p_{2}\left(g_{1}(x)\right)=100+0.07 g_{1}(x) \mathrm{K} €$, respectively, where $x$ is the worst of the two actions (c.f. Section 3.3).

- The evaluations of criteria $g_{3}$ (recruitment), $g_{4}$ (image), and $g_{5}$ (access) are expressed on the following seven-level qualitative scale: very bad (1), bad (2), rather bad (3), average (5), rather good (5), good (6), and very good (7). The values between parenthesis can be used in ELECTRE methods to code the different verbal statements. Let us notice that such a way of coding plays only a purely ordinal role in the computation; other ways of coding the verbal scale trough the use of numerical values could be used by adjusting the thresholds values (see Martel and Roy, 2006).The indifference threshold for each criterion has been set at one on the seven-level scale and the preference threshold at two levels.

\begin{tabular}{|c|c|c|c|c|c|}
\hline & $g_{1}[\mathrm{~min}]$ & $g_{2}[\mathrm{~min}]$ & $g_{3}[\mathrm{max}]$ & $g_{4}[\mathrm{max}]$ & $g_{5}[\mathrm{max}]$ \\
\hline$a$ & $13000 \mathrm{~K} €$ & $3000 \mathrm{~K} €$ & Average & Average & Average \\
\hline$b$ & $15000 \mathrm{~K} €$ & $2500 \mathrm{~K} €$ & Good & Bad & Very Good \\
\hline$c$ & $10900 \mathrm{~K} €$ & $3400 \mathrm{~K} €$ & Good & Good & Very Bad \\
\hline$d$ & $15500 \mathrm{~K} €$ & $3500 \mathrm{~K} €$ & Good & Good & Good \\
\hline$e$ & $15000 \mathrm{~K} €$ & $2600 \mathrm{~K} €$ & Good & Very Bad & Bad \\
\hline
\end{tabular}

Table 4: Some potential sites for the new hotel

Consider again the weights obtained using SRF, $k_{1}=5, k_{2}=4, k_{3}=k_{4}=k_{5}=3$, where $K=18$. The concordance index for the ordered pair $(a, d)$ is $c(a, d)=\frac{(5+4)}{18}=\frac{1}{2}$. Taking into account the mutual strengthening interaction effect between $g_{1}$ and $g_{2}$, whose the value is set at $k_{12}=3$ as defined in Section 4.2, our normalization coefficient $K(a, d)=$ $18+3=21$. The new $c(a, d)=\frac{12}{21}=\frac{4}{7}$. In fact, $c(d, a)$ does not change whether or not the interaction coefficient $k_{12}$ is taken into account (i.e., $c(d, a)=\frac{9}{18}=\frac{1}{2}$ ). If the concordance threshold $s$ has been defined as $s=0.55$, the mutual strengthening interaction effect makes it clear that site $a$ is better than $d$, whereas they were previously incomparable.

For the comparison between sites $a$ and $e, c(a, e)=\frac{(5+3+3)}{18}=\frac{11}{18}$. But, when considering the mutual weakening interaction effect modeled with $k_{45}=-2, K(a, e)=18-2=16$ and $c(a, e)=\frac{(5+3+3-2)}{16}=\frac{9}{16}$, the concordance index $c(e, a)$ takes always the same value: $c(e, a)=\frac{(4+3)}{18}=\frac{7}{18}$. For $s=0.55, a$ can no longer be compared to $e$, whereas it was the preferred site prior to applying the mutual weakening effect.

\subsection{Launching a new digital camera model}

Table 5 presents the evaluations of the four models - $a, b, c$, and $d$ - according to the seven criteria given in Section 2. Let us precise that:

- the evaluations of criterion $g_{1}$ (price) are expressed in $€$; the indifference and the preference thresholds assigned to this criterion are $q_{1}=25 €$ and $p_{1}=50 €$, respectively;

- the evaluations of criterion $g_{6}$ (volume) are expressed in cubic centimeters; the thresholds assigned to this criterion are $q_{6}=10 \mathrm{~cm}^{3}$ and $p_{6}=20 \mathrm{~cm}^{3}$, respectively;

- the evaluations of criterion $g_{7}$ (weight) are expressed in grams; the thresholds are $q_{7}=10 \mathrm{~g}$ and $p_{7}=20 \mathrm{~g}$, respectively;

- the evaluations of criteria $g_{2}$ (weakness), $g_{3}$ (workability), $g_{4}$ (image), and $g_{5}$ (aesthetics) are expressed on the following seven-level qualitative scale: very bad (1), bad (2), rather bad (3), average (4), rather good (5), good (6), and very good (7); these criteria have an indifference threshold of one on the seven-level scale and a preference threshold of two. 


\begin{tabular}{|c|c|c|c|c|c|c|c|}
\hline & $g_{1}[\mathrm{~min}]$ & $g_{2}[\mathrm{~min}]$ & $g_{3}[\mathrm{max}]$ & $g_{4}[\mathrm{max}]$ & $g_{5}[\mathrm{max}]$ & $g_{6}[\mathrm{~min}]$ & $g_{7}[\mathrm{~min}]$ \\
\hline$a$ & $220 €$ & Average & Average & Rather Good & Average & $190 \mathrm{~cm}^{3}$ & $155 \mathrm{~g}$ \\
\hline$b$ & $300 €$ & Bad & Rather Good & Average & Rather Good & $160 \mathrm{~cm}^{3}$ & $145 \mathrm{~g}$ \\
\hline$c$ & $160 €$ & Bad & Very Bad & Average & Rather Bad & $140 \mathrm{~cm}^{3}$ & $130 \mathrm{~g}$ \\
\hline$d$ & $280 €$ & Very Good & Average & Very Good & Average & $220 \mathrm{~cm}^{3}$ & $170 \mathrm{~g}$ \\
\hline
\end{tabular}

Table 5: Some possible digital camera models

Consider again the weights obtained by using SRF, $k_{1}=6, k_{2}=4, k_{3}=k_{4}=k_{5}=1, k_{6}=k_{7}=2$, where $K=17$. The concordance index for $(a, d)$ is $c(a, d)=\frac{(6+1+1+2+1)}{17}=\frac{11}{17}$ (criterion $g_{7}$ is in the ambiguity zone, and it only counts for $50 \%$ of its overall weight). Now, consider the antagonistic effect, where $k_{12}^{\prime}=2.5$. The new concordance index takes the value $c(a, d)=\frac{8.5}{14.5}$. But, $c(d, a)$ remains the same (i.e., $\left.c(d, a)=\frac{(4+3+1)}{17}=\frac{8}{17}\right)$. If $s$ is defined at $s=0.6$, when taking the antagonistic effect into account, the actions become incomparable, although $a$ was preferred to $d$ before. This incomparability shows that this effect can imply significant changes.

\section{Concordance index and the Choquet integral}

The Choquet integral (see Choquet, 1953) is an aggregation operator permitting to model interactions between criteria. It is used to build a value function giving a complete preorder, i.e., a transitive and strongly complete binary relation, rather than simply an outranking relation, being only reflexive and not transitive and complete, as it is the case in ELECTRE type methods. Moreover, the way in which the Choquet integral is used is questionable especially with respect to two main points as stated by Roy (2007):

1. the hypothesis that the evaluation of each criterion is supposed to be expressed on the same scale in a meaningful way; and,

2. the way in which the importance of criteria are measured through the Shapley indices.

In what follows we will show that the numerator of the new concordance index of formula (6) can be interpreted as the classical Choquet integral under two conditions: no antagonistic effect is taken into account, and $Z=\min \{x, y\}$. Finally, we will show that for modeling the numerator of the new concordance index of formula (6) in case of antagonistic effect we need to use the bipolar Choquet integral.

The Choquet integral (see Choquet, 1953) of a vector $x=\left(x_{1}, x_{2}, \ldots, x_{n}\right) \in \mathbb{R}_{+}^{n}$ with respect to a capacity $\mu$ being a function $\mu: 2^{F} \rightarrow[0,1]$, such that

1. $\mu(B) \geq \mu(C)$, for all $B \subseteq C \subseteq F$

2. $\mu(\varnothing)=0$ and $\mu(F)=1$,

is defined

$$
C h(x, \mu)=\sum_{i=1}^{n}\left(x_{(i)}-x_{(i-1)}\right) \mu\left(B_{(i)}\right)
$$

where, $(\cdot)$ indicates a permutation of $F$ such that $x_{(1)} \leq x_{(2)} \leq \ldots \leq x_{(n)}, x_{(0)}=0$ and $B(i)=\{(i), \ldots,(n)\}$. The Choquet integral can be interpreted as a generalization of the weighted average aggregation method when interactions between criteria have to be taken into account. This is clear understandable after the concept of Möbius transform is introduced and the Choquet integral is reformulated according to such a transform. Given a capacity $\mu$, its Möbius transform (see, for example Rota, 1964) is given by the values $a(S) \in \mathbb{R}, S \subseteq F$, such that

$$
a(S)=\sum_{T \subseteq S}(-1)^{|S-T|} \mu(T), S \subseteq F
$$

Using the Möbius transform, the capacity can be expressed as

$$
\mu(S)=\sum_{T \subseteq S} a(T), S \subseteq F
$$


while the Choquet integral can be rewritten as follows,

$$
C h(x, \mu)=\sum_{T \subseteq S} a(T) \min \left\{x_{i}: i \in T\right\}
$$

Let us remark that the values of $a(S), S \subseteq F$, are related to the interaction of elements from $S$. Thus if there is no interaction, we have $a(S)=0$ for all $S \subseteq F$ with $|S|>1$, and thus,

$$
\mu(S)=\sum_{i \in A} a(\{i\}), S \subseteq F
$$

while the Choquet integral becomes,

$$
C h(x, \mu)=\sum_{i \in F} a(\{i\}) x_{i}=\sum_{i \in F} \mu(\{i\}) x_{i}
$$

that is the Choquet integral collapses to the weighted average method of values $x_{i}$ with weights $\mu(\{i\})=a(\{i\})$. An interesting case of interaction, often used in the applications of Choquet integral for its simplicity, is given by 2-additive capacity (see Grabisch, 1996), being a capacity $\mu$ such that for its Möbius transform we have that $a(S)=0$ for all $S \subseteq F$ with $|S|>2$, and thus

$$
\mu(S)=\sum_{i \in S} a(\{i\})+\sum_{\{i, j\} \subseteq S} a(\{i, j\}), S \subseteq F
$$

while the Choquet integral becomes,

$$
C h(x, \mu)=\sum_{i \in F} a(\{i\}) x_{i}+\sum_{\{i, j\} \subseteq F} a(\{i, j\}) \min \left\{x_{i}, x_{j}\right\} .
$$

Looking at the concordance index from the point of view of Choquet integral (since in case of absence of interactions the concordance index of ELECTRE methods is the weighted average of values $c_{i}(a, b)$ ), it can be seen as the Choquet integral of values $c_{i}(a, b)$ with a capacity $\mu(S)=\frac{\sum_{i \in S} k_{i}}{K}$ for all $S \subseteq F$. Instead, in case of presence of mutual strengthening or mutual weakening effect, but not the antagonistic effect, then the numerator of the concordance index we proposed in the previous sections corresponds to the Choquet integral of values $c_{i}(a, b)$ with a capacity $\mu(S)=\sum_{i \in S} k_{i}+\sum_{\{i, j\} \subseteq S} k_{i j}$, for all $S \subseteq F$ in case of $Z(x, y)=\min \{x, y\}$.

The antagonistic effect cannot be taken into account with the above formula. As for taking it into account we will consider the bipolar Choquet integral.

Given the set or family of criteria, $F=\left\{g_{1}, g_{2}, \ldots, g_{i}, \ldots, g_{n}\right\}$ or simply $F=\{1,2, \ldots, i, \ldots, n\}$ consider the set $M=\{(B, C): B, C \subseteq F, B \cap C \neq \varnothing\}$.

The antagonistic effect can be modeled in the framework of the bipolar Choquet integral (see Grabisch and Labreuche, 2005a and Greco et al., 2002). A bicapacity (Grabisch and Labreuche, 2005a, 2005b) is a function $\mu_{b}: M \rightarrow[-1,1]$ such that,

1) for all $B \subseteq D \subseteq F$ and $E \subseteq C \subseteq F$ such that $(B, C),(D, E) \in M, \mu_{b}(A, B) \leq \mu_{b}(C, D)$,

2) $\mu_{b}(\varnothing, \varnothing)=0$,

3) $\mu_{b}(F, \varnothing)=1$ and $\mu_{b}(\varnothing, F)=-1$.

A bipolar capacity (Greco et al, 2002) is a function

$$
\mu_{b i p}: M \rightarrow[0,1] \times[0,1], \quad(B, C) \rightarrow \mu_{b i p}(B, C)=\left(\mu_{b i p}^{+}(B, C), \mu_{b i p}^{-}(B, C)\right)
$$

such that,

4) for all $B \subseteq D \subseteq F$ and $E \subseteq C \subseteq F$ such that $(B, C),(D, E) \in M, \mu_{b i p}^{+}(B, C) \leq \mu_{b i p}^{+}(D, E)$ and $\mu_{b i p}^{-}(B, C) \geq \mu_{b i p}^{-}(D, E)$,

5) for all $B \subseteq F, \mu_{b i p}^{+}(\varnothing, B)=0$ and $\mu_{b i p}^{-}(B, \varnothing)=0$, 
6) $\mu_{b i p}^{+}(F, \varnothing)=1$ and $\mu_{b i p}^{-}(\varnothing, F)=1$.

Now, the bipolar Choquet integral $\left(C h_{b}\right)$ of $x \in \mathbb{R}^{n}$, with respect to bicapacity $\mu_{b}$, can be defined as follows (Grabisch and Labreuche, 2005b),

$$
C h_{b}\left(x, \mu_{b}\right)=\sum_{i=1}^{n}\left(\left|x_{(i)}\right|-\left|x_{(i-1)}\right|\right) \mu_{b}\left(B_{(i)}^{+}, B_{(i)}^{-}\right)
$$

where, [.] indicates a permutation of $F$ such that $\left|x_{(1)}\right|,\left|x_{(2)}\right|, \leq, \ldots, \leq\left|x_{(n)}\right|,\left|x_{(0)}\right|=0, A_{(i)}^{+}=\left\{j \in F: x_{j} \geq\left|x_{i}\right|\right\}$, and $A_{(i)}^{-}=\left\{j \in F: x_{j}<0,-x_{j} \geq\left|x_{i}\right|\right\}$.

And, the bipolar Choquet integral $\left(C h_{b i p}\right)$ of $x \in \mathbb{R}^{n}$, with respect to bipolar capacity $\mu_{b i p}$, can be defined as follows,

$$
C h_{b i p}\left(x, \mu_{b i p}\right)=C h_{b i p}^{+}\left(x, \mu_{b i p}\right)-C h_{b i p}^{-}\left(x, \mu_{b i p}\right)
$$

with

$$
C h_{b i p}^{+}\left(x, \mu_{b i p}\right)=\sum_{i=1}^{n}\left(\left|x_{(i)}\right|-\left|x_{(i-1)}\right|\right) \mu_{b i p}^{+}\left(B_{(i)}^{+}, B_{(i)}^{-}\right)
$$

being the positive component of the bipolar Choquet integral, and

$$
C h_{b i p}^{-}\left(x, \mu_{b i p}\right)=\sum_{i=1}^{n}\left(\left|x_{(i)}\right|-\left|x_{(i-1)}\right|\right) \mu_{b i p}^{-}\left(B_{(i)}^{+}, B_{(i)}^{-}\right)
$$

being the negative component of the bipolar Choquet integral (Greco et al, 2002).

To calculate the bipolar Choquet integral we have to fix the value of $\mu_{b}(B, C)$ for all $(B, C) \in M$, while to calculate the positive and the negative components of the bipolar Choquet integral we have to fix the value of $\mu_{b i p}^{+}(B, C)$ and $\mu_{b i p}^{-}(B, C)$ for all $(B, C) \in M$. Thus, to apply the bipolar Choquet integral a very large number of parameters should be defined. To deal with this problem Grabisch and Labreuche (2005a) proposed the 2-additive bicapacities, while Greco and Figueira (2003) proposed the 2-order decomposable bipolar capacities. The 2-order decomposable bipolar capacity measure gives us a model to compare the bipolar Choquet integral with the concordance index in case where the antagonistic effect is present.

A bipolar capacity is 2-order decomposable if there exists, $a^{+}(\{j\}, \varnothing), a^{+}(\{j, k\}, \varnothing), a^{+}(\{j\},\{k\}), a^{-}(\varnothing,\{j\})$, $a^{-}(\varnothing,\{j, k\}), a^{-}(\{j\},\{k\}) \in \mathbb{R}, j, k \in F, j \neq k$, such that, for all $(B, C) \in M$,

$$
\begin{aligned}
& \text { - } \mu_{\text {bip }}^{+}(B, C)=\sum_{j \in B} a^{+}(\{j\}, \varnothing)+\sum_{j, k \in B} a^{+}(\{j, k\}, \varnothing)+\sum_{j \in B, k \in C} a^{+}(\{j\},\{k\}) \\
& \text { - } \mu_{b i p}^{-}(B, C)=\sum_{j \in B} a^{-}(\varnothing,\{j\})+\sum_{j, k \in B} a^{-}(\varnothing,\{j, k\})+\sum_{j \in B, k \in C} a^{-}(\{j\},\{k\})
\end{aligned}
$$

The bipolar Choquet integral $\left(C h_{b i p}\right)$ of $x \in \mathbb{R}^{n}$, with respect to a 2-order decomposable bipolar capacity $\mu_{b i p}$, can be defined as follows,

$$
C h_{b i p}\left(x, \mu_{b i p}\right)=C h_{b i p}^{+}\left(x, \mu_{b i p}\right)-C h_{b i p}^{-}\left(x, \mu_{b i p}\right)
$$

with,

$$
\begin{aligned}
C h_{\text {bip }}^{+}\left(x, \mu_{\text {bip }}\right)= & \sum_{j \in F, x_{j}>0} a^{+}(\{j\}, \varnothing) x_{j}+\sum_{j, k \in F, x_{j}, x_{k}>0} a^{+}(\{j, k\}, \varnothing) \min \left\{x_{j}, x_{k}\right\}+ \\
& +\sum_{j, k \in F, x_{j}>0, x_{k}<0} a^{+}(\{j\},\{k\}) \min \left\{x_{j},-x_{k}\right\}
\end{aligned}
$$

being the positive component of the bipolar Choquet integral, and

$$
\begin{aligned}
C h_{\text {bip }}^{-}\left(x, \mu_{\text {bip }}\right)= & \sum_{j \in F, x_{j}<0} a^{-}(\varnothing,\{j\})\left(-x_{j}\right)+\sum_{j, k \in F, x_{j}, x_{k}<0} a^{-}(\varnothing,\{j, k\}) \min \left\{-x_{j},-x_{k}\right\}+ \\
& +\sum_{j, k \in F, x_{j}>0, x_{k}<0} a^{-}(\{j\},\{k\}) \min \left\{x_{j},-x_{k}\right\}
\end{aligned}
$$


being the negative component of the bipolar Choquet integral (Greco and Figueira, 2003).

Observe that the numerator of the concordance index we propose, in case of $Z(x, y)=\min \{x, y\}$, corresponds to the positive part of the bipolar Choquet integral of vector $x=\left(x_{1}, \ldots, x_{n}\right)$ with $x_{i}=c_{i}(a, b)$ if $i \in \bar{C}(b P a)$ and $x_{i}=-c_{i}(b, a)=$ -1 if $i \in C(b P a)$ in case

$$
\mu_{\text {bip }}^{+}(R, S)=\sum_{i \in S} k_{i}+\sum_{\{i, j\} \subseteq S} k_{i j}+\sum_{i \in S, h \in R} k_{i h}^{\prime}, \text { for all }(R, S) \in M,
$$

which proves the relation between our proposal and Choquet integral for this particular case.

\section{Conclusion}

In this paper we introduced three types of interaction that allow modeling a large number of dependence situations in real-world decision-aiding problems. We showed how to take into account these types of interaction in the concordance index used within the ELECTRE methods framework. For this purpose, formula (2) can be simply replaced by (6) in all of the ELECTRE methods. We explained how the extension of the concordance index we are proposing can be used in practice. Nevertheless, this extension is appropriate only when the number of pairs of interaction criteria is rather small. Otherwise, we considere that the family of criteria should be rebuilt, since it contains too many interactions and possibly incoherencies. In addition, we showed the links between our approach and the Choquet integral. As a line for possible investigation in the future we can mention the study of the interactive protocol of the decision-makers or their representatives when facing to situations with interaction between criteria in real-world problems. A software development and implementation will also be one of the main concerns in the near future.

Acknowledgements The first and the third authors acknowledge the support from Luso-French PESSOA bilateral cooperation. The authors acknowledges Benedetto Matarazzo and Manuel Matos for the valuable comments, remarks, and suggestions they made on a draft version of this paper. This research also partially benefited from the COST Action 0602 research project on "Algorithmic Decision Theory".

\section{Appendix}

Proof of Lemma 1.

The proof of this lemma is based on the fact that, if the difference $\Delta_{f}$ decreases, either $S(a, b)$ remains constant or it decreases. Two cases should be considered.

1. Criterion $f$ belongs to $C(b P a)$.

If $f$ belongs to $C(b P a)$ it cannot belong to $\bar{C}(b P a)$. Consequently, the pair $\{i, f\}$ will not belong to $L(a, b)$. The decreasing of $\Delta_{f}$ does not affect neither the first nor the second summations in the formula of $S(a, b)$. Whatever, it will occur with the existence or not of ordered pairs $(i, f) \in O(a, b)$, the decreasing of $\Delta_{f}$ has no influence on the third summation. Consequently, $S(a, b)$ remains constant.

2. Criterion $f$ belongs to $\bar{C}(b P a)$.

Two subcases have to be considered,

(a) Criterion $f$ stills remain in $\bar{C}(b P a)$.

The decreasing of $\Delta_{f}$ will not move $f$ from $\bar{C}(b P a)$. Hence, the three summations in the definition of $S(a, b)$ will not be affected. Then, $S(a, b)$ remains constant too.

(b) Criterion $f$ moves to $C(b P a)$.

The decreasing of $\Delta_{f}$ moves $f$ from $\bar{C}(b P a)$ to $C(b P a)$. This moving has some implications on the result. The new value of $S(a, b)$ will become,

$$
S(a, b)^{\text {New }}=S(a, b)^{\text {Old }}-\left(k_{f}+\sum_{\{f, j\} \in L^{\text {Old }}(a, b)} k_{f j}-\sum_{(f, h) \in O^{\text {Old }}(a, b)} k_{f h}^{\prime}+\sum_{(i, f) \in O^{\text {New }}(a, b)} k_{i f}^{\prime}\right)
$$

where, $L^{\text {Old }}(a, b), O^{\text {Old }}(a, b)$ represent the sets $L(a, b)$ and $O(a, b)$ before $\Delta_{f}$ decreases and $O^{\text {New }}(a, b)$ represents the set $O(a, b)$ after $\Delta_{f}$ decreases. The quantity in between big parenthesis is necessarily non-negative according to the net balance condition, which ensures

$$
k_{f}+\sum_{\{f, j\} \in L_{\text {old }}(a, b)} k_{f j}-\sum_{(f, h) \in O^{\text {old }}(a, b)} k_{f h}^{\prime}>0,
$$


and the positivity of the parameters relative to the antagonism effect, which ensures

$$
\sum_{(i, f) \in O^{\text {New }}(a, b)} k_{i f}^{\prime} \geq 0
$$

Consequently, $S(a, b)$ cannot increase.

The proof of the monotonicity of $S(a, b)$ is complete. Let us now show that $S(a, b) \geq 0$.

If $\bar{C}(b P a)=\varnothing$, then $S(a, b)=0$. Suppose that we could have $S(a, b)<0$. This implies that at least one criterion $f$ does not belong to $\bar{C}(b P a) \neq \varnothing$. Consider that there exists at least one criterion in $\bar{C}(b P a)$. If for all $f$ in $\bar{C}(b P a), \Delta_{f}$ is forced to decrease till $\bar{C}(b P a)=\varnothing$, then $S(a, b)$ cannot increase. Contradiction!

The proof is now complete.

\section{Proof of Lemma 2.}

The proof of this lemma is based on the fact that, if the difference $\Delta_{f}$ decreases, either $c(a, b)$ remains constant or it decreases. Two cases should be considered.

1. Criterion $f$ belongs to $C(b P a)$.

If $f$ belongs to $C(b P a)$, after $\Delta_{f}$ decreases it continues to belong to $C(b P a)$, such that the sets $\bar{C}(b P a), L(a, b)$ and $O(a, b)$ remain unchanged. Consequently, the value of $K(a, b)$ and the three summations in (4) do not change and, therefore, $c(a, b)$ remains constant.

2. Criterion $f$ belongs to $\bar{C}(b P a)$.

Two subcases have to be considered,

(a) Criterion $f$ stills remain in $\bar{C}(b P a)$.

Decreasing $\Delta_{f}$ will not make a move of $f$ from $\bar{C}(b P a)$ to the opposite coalition $C(b P a)$. Again, the value of $K(a, b)$ does not change. Hence, the three summations will not be affected. Then, $c(a, b)$ remains constant too.

(b) Criterion $f$ moves to $C(b P a)$.

When decreasing $\Delta_{f}, f$ moves from $\bar{C}(b P a)$ to $C(b P a)$. This moving has some implications on the result. Consider the following additional notation,

$$
\begin{aligned}
& \alpha=S(a, b)^{\text {New }} \\
& \beta=K(a, b)^{\text {New }} \\
& \gamma=K(a, b)^{\text {Old }}-K(a, b)^{\text {New }} \\
& \delta=k_{f}
\end{aligned}
$$

where $K(a, b)^{\text {Old }}$ denotes the value of $K(a, b)$ before $\Delta_{f}$ decreases, and $S(a, b)^{\text {New }}$ and $K(a, b)^{\text {New }}$ denote the value of $S(a, b)$ and $K(a, b)$ after $\Delta_{f}$ decreases.

The concordance indices $c(a, b)^{\text {Old }}$ and $c(a, b)^{\text {New }}$ can be rewritten as follows,

$$
c(a, b)^{\text {Old }}=\frac{\alpha+\gamma+\delta}{\beta+\gamma}
$$

and

$$
c(a, b)^{N e w}=\frac{\alpha}{\beta}
$$

Therefore, the monotonicity condition $c(a, b)^{\text {Old }}>c(a, b)^{\text {New }}$ becomes $\frac{\alpha+\gamma+\delta}{\beta+\gamma}>\frac{\alpha}{\beta}$. Since $\beta=K(a, b)^{\text {New }}$ and $\beta+\gamma=K(a, b)^{\text {Old }}$, we have $\beta>0$ and $\beta+\gamma>0$. Through the application of simple algebraic operations, we get,

$$
\frac{\alpha+\gamma+\delta}{\beta+\gamma}>\frac{\alpha}{\beta} \Leftrightarrow(\alpha+\gamma+\delta) \beta>(\beta+\gamma) \alpha \Leftrightarrow(\gamma+\delta) \beta>\alpha \gamma
$$

Two cases are possible:

- $\gamma \leq 0$ : in this case $(\gamma+\delta) \beta>0$ (observe that, by lemma $1, \gamma+\delta=S(a, b)^{\text {Old }}-S(a, b)^{\text {New }}>0$ ) and $\alpha \gamma \leq 0$ (notice that $\alpha \geq 0$, because $\alpha$ equals $S(a, b)$ after decreasing $\Delta_{f}$ and, by Lemma $1, S(a, b)$ is always non-negative),

- $\gamma>0$ : since $\beta>\alpha$ (notice that $\beta-\alpha=\sum_{i \in C(b P a)} k_{i}$ ) and $\alpha \geq 0$, (i) holds if $\delta>0$, which is always true.

Therefore, in any case $(i)$ is true and we can conclude that, when decreasing $\Delta_{f}, c(a, b)$ also decreases.

The proof is complete. 
Proof of Lemma 3.

The proof of this lemma is also based on the fact that if the difference $\Delta_{f}$ decreases, either $S(a, b)$ remains constant or it decreases. Two cases have to be considered.

1. Criterion $f$ belongs to $C(b P a)$.

As in the absence of pseudo-criteria, after $\Delta_{f}$ decreases it continues to belong to $C(b P a)$, such that the sets $\bar{C}(b P a), L(a, b)$ and $O(a, b)$ remain unchanged. Moreover, if there exist ordered pairs $(i, f) \in O(a, b)$, then when decreasing $\Delta_{f} c_{f}(b, a)$ remains equal to 1 . Consequently, the value of $K(a, b)$ and the three summations in (6) do not change and, therefore, $c(a, b)$ remains constant.

2. Criterion $f$ belongs to $\bar{C}(b P a)$.

Now, three subcases have to be considered.

(a) Criterion $f$ belongs to $C(a S b)$.

The decreasing of $\Delta_{f}$ does not moves $f$; it remains thus in $C(a S b)$. More precisely, the decreasing of $\Delta_{f}$ will not make any change in the three components of $S(a, b)$, which remains constant.

(b) Criterion $f$ belongs to $C(b Q a)$.

After decreasing $\Delta_{f}$, criterion $f$ stills remain in $C(b Q a)$, either because it belonged to this coalition before or because it moved to $C(b Q a)$ due to the decreasing of $\Delta_{f}$. All the summations in the definition of $S(a, b)$ are affected. Let us suppose that $c_{f}(a, b)$ changes its new value and becomes $c_{f}(a, b)-\Delta$, with $\Delta>0$. We have the following inequality,

$$
\begin{gathered}
S(a, b)^{N e w}-S(a, b)^{\text {Old }}= \\
-\Delta k_{f}+\sum_{j \in \bar{C}(a, b)}\left(Z\left(c_{f}(a, b)-\Delta, c_{j}(a, b)\right)-Z\left(c_{f}(a, b), c_{j}(a, b)\right)\right) k_{f j}+ \\
-\sum_{h \in C(b P a)}\left(Z\left(c_{f}(a, b)-\Delta, c_{h}(b, a)\right)-Z\left(c_{f}(a, b), c_{h}(b, a)\right)\right) k_{f h}^{\prime} \\
\leq \\
-\Delta k_{f}-\sum_{j \in \bar{C}(a, b): k_{f j}<0}\left(Z\left(c_{f}(a, b)-\Delta, c_{j}(a, b)\right)-Z\left(c_{f}(a, b), c_{j}(a, b)\right)\right)\left|k_{f j}\right|+ \\
-\sum_{h \in C(b P a)}\left(Z\left(c_{f}(a, b)-\Delta, c_{h}(b, a)\right)-Z\left(c_{f}(a, b), c_{h}(b, a)\right)\right) k_{f h}^{\prime}
\end{gathered}
$$

Let us remark that for all $h \in C(b P a)$,

$$
Z\left(c_{f}(a, b)-\Delta, c_{h}(b, a)\right)-Z\left(c_{f}(a, b), c_{h}(b, a)\right)=-\left(Z\left(c_{f}(a, b), c_{h}(b, a)\right)-Z\left(c_{f}(a, b)-\Delta, c_{h}(b, a)\right)\right)
$$

From the marginal impact condition and putting $c_{f}(a, b)-\Delta=x, c_{h}(b, a)=y$, and $\Delta=w$ in the previous condition we obtain,

$$
\frac{1}{\Delta}\left(Z\left(c_{f}(a, b), c_{h}(b, a)\right)-Z\left(c_{f}(a, b)-\Delta, c_{h}(b, a)\right)\right) \leq 1
$$

and therefore,

$$
Z\left(c_{f}(a, b), c_{h}(b, a)\right)-Z\left(c_{f}(a, b)-\Delta, c_{h}(b, a)\right) \leq \Delta
$$

or, in an equivalent way,

$$
Z\left(c_{f}(a, b)-\Delta, c_{h}(b, a)\right)-Z\left(c_{f}(a, b), c_{h}(b, a)\right) \geq-\Delta
$$

And, now from this expression we obtain,

$$
\begin{aligned}
-\Delta k_{f}- & \sum_{j \in \bar{C}(a, b): k_{f j}<0}\left(Z\left(c_{f}(a, b)-\Delta, c_{j}(a, b)\right)-Z\left(c_{f}(a, b), c_{j}(a, b)\right)\right)\left|k_{f j}\right|+ \\
& -\sum_{h \in C(b P a)}\left(Z\left(c_{f}(a, b)-\Delta, c_{h}(b, a)\right)-Z\left(c_{f}(a, b), c_{h}(b, a)\right)\right) k_{f h}^{\prime}
\end{aligned}
$$




$$
-\Delta k_{f}+\Delta \sum_{j \in \bar{C}(a, b): k_{f j}<0}\left|k_{f j}\right|+\Delta \sum_{h \in C(b P a)} k_{f h}^{\prime}=-\Delta\left(k_{f}-\sum_{j \in \bar{C}(a, b): k_{f j}<0}\left|k_{f j}\right|-\sum_{h \in C(b P a)} k_{f h}^{\prime}\right)
$$

From the net balance condition we have,

$$
k_{f}-\sum_{j \in \bar{C}(a, b): k_{f j}<0}\left|k_{f j}\right|-\sum_{h \in C(b P a)} k_{f h}^{\prime}>0
$$

and, therefore, since $\Delta>0$, we have

$$
-\Delta\left(k_{f}-\sum_{j \in \bar{C}(a, b): k_{f j}<0}\left|k_{f j}\right|-\sum_{h \in C(b P a)} k_{f h}^{\prime}\right)<0
$$

such that

$$
S(a, b)^{\text {New }}-S(a, b)^{\text {Old }}<0
$$

and, we can conclude that, after decreasing $\Delta_{f}, S(a, b)$ decreases.

(c) Criterion $f$ moves to $C(b P a)$.

The decreasing of $\Delta_{f}$ will move $f$ to $C(b P a)$. In such a case, $c_{f}(a, b) k_{f}$ can no more be found in the expression of $S(a, b)$. If there are $j$ such that $\{f, j\} \in L(a, b)$, then the terms $Z\left(c_{f}(a, b), c_{j}(a, b)\right) k_{f j}$ will be removed from the second summation of (6). For all $i \in \bar{C}(b P a)$, the pair $(i, f)$ enters in $O(a, b)$, while for all $h \in C(b P a)$ the pair $(f, h)$ goes out from $O(a, b)$. The new value of $S(a, b)$, $S(a, b)^{N e w}$, is equal to the previous one, $S(a, b)^{\text {Old }}$, minus a certain quantity; it is calculated as follows,

$$
\begin{gathered}
S(a, b)^{N e w}=S(a, b)^{\text {Old }} \\
-\left(c_{f}(a, b) k_{f}+\sum_{\{f, j\} \in L(a, b)} Z\left(c_{f}(a, b), c_{j}(a, b)\right) k_{f j}\right. \\
\left.-\sum_{(f, h), h \in C(b P a)} Z\left(c_{f}(a, b), c_{h}(b, a)\right) k_{i f}^{\prime}+\sum_{(i, f), i \in \bar{C}(b P a)} Z\left(c_{i}(a, b), c_{f}(b, a)\right) k_{i f}^{\prime}\right)
\end{gathered}
$$

Now, we have to prove that the quantity between parentheses, denoted by $\Delta S(a, b)$, is non-negative. Remembering that $Z(x, y) \leq$ $\min \{x, y\}$ for all $x, y \in[0,1]$ and the net balance condition we get

$$
\begin{gathered}
\Delta S(a, b)=c_{f}(a, b) k_{f}+\sum_{\{f, j\} \in L(a, b)} Z\left(c_{f}(a, b), c_{j}(a, b)\right) k_{f j} \\
-\sum_{(f, h), h \in C(b P a)} Z\left(c_{f}(a, b), c_{h}(b, a)\right) k_{f h}^{\prime}+\sum_{(i, f), i \in \bar{C}(b P a)} Z\left(c_{i}(a, b), c_{f}(b, a)\right) k_{i f}^{\prime} \\
c_{f}(a, b) k_{f}-\sum_{\{f, j\} \in L(a, b), k_{f j}<0} Z\left(c_{f}(a, b), c_{j}(a, b)\right)\left|k_{f j}\right| \\
-\sum_{(f, h), h \in C(b P a)} Z\left(c_{f}(a, b), c_{h}(b, a)\right) k_{f h}^{\prime}+\sum_{(i, f), i \in \bar{C}(b P a)} Z\left(c_{i}(a, b), c_{f}(b, a)\right) k_{i f}^{\prime} \\
c_{f}(a, b)\left(k_{f}-\sum_{\{f, j\} \in L(a, b), k_{f j}<0}\left|k_{f j}\right|-\sum_{(f, h), h \in C(b P a)} k_{f h}^{\prime}\right) \\
+\sum_{(i, f), i \in \bar{C}(b P a)} Z\left(c_{i}(a, b), c_{f}(b, a)\right) k_{i f}^{\prime}>0
\end{gathered}
$$

Consequently, $S(a, b)$ decreases.

The proof of the monotonicity of $S(a, b)$ is thus complete. The proof of $S(a, b) \geq 0$ can be obtained from the monotonicity of $S(a, b)$ in an analogous way as in the case of quasi criteria. 
Proof of Lemma 4.

The proof of this lemma is also based on the fact that if the difference $\Delta_{f}$ decreases, either $c(a, b)$ remains constant or it decreases. Two cases have to be considered.

1. Criterion $f$ belongs to $C(b P a)$.

For the same reasons as in the absence of pseudo-criteria, $c(a, b)$ remains constant.

2. Criterion $f$ belongs to $\bar{C}(b P a)$.

Now, four subcases have to be considered.

(a) Criterion $f$ belongs to $C(a S b)$ and it remains in $C(a S b)$ after decreasing $\Delta_{f}$.

The decreasing of $\Delta_{f}$ does not move $f$ to another coalition. Thus, the decreasing of $\Delta_{f}$ will not make any change in the three components of the numerator of $c(a, b)$, neither in the normalization coefficient $K(a, b)$. Consequently, $c(a, b)$ remains constant.

(b) Criterion $f$ moves from $C(a S b)$ to $C(b Q a)$.

After decreasing $\Delta_{f}$, criterion $f$ moves to $C(b Q a)$ due to this decreasing and the value of $c_{f}(a, b)$ passes from 1 to $c_{f}(a, b)^{\text {New }}<1$. All the summations in the numerator of (6) are affected. The second and the third summation in the denominators can be modified also.

Consider the following additional notation,

$$
\begin{aligned}
& \alpha=S(a, b)^{\text {New }} \\
& \beta=K(a, b)^{\text {New }} \\
& \gamma=K(a, b)^{\text {Old }}-K(a, b)^{\text {New }} \\
& \delta=k_{f}\left(1-c_{f}(a, b)^{\text {New }}\right)
\end{aligned}
$$

where $K(a, b)^{\text {Old }}$ denotes the value of $K(a, b)$ before $\Delta_{f}$ decreases, and $S(a, b)^{\text {New }}$ and $K(a, b)^{\text {New }}$ denote the value of $S(a, b)$ and $K(a, b)$ after $\Delta_{f}$ decreases.

Thus, we have that

$$
c(a, b)^{\text {Old }}=\frac{\alpha+\gamma+\delta}{\beta+\gamma} \text { and } c(a, b)^{\text {New }}=\frac{\alpha}{\beta}
$$

such that the monotonicity condition $c(a, b)^{\text {Old }}>c(a, b)^{N e w}$ becomes as follows,

$$
\frac{\alpha+\gamma+\delta}{\beta+\gamma}>\frac{\alpha}{\beta}
$$

Observe that $\beta>0$ and $\beta+\gamma>0$ (because $\beta=K(a, b)^{\text {New }}$ and $\beta+\gamma=K(a, b)^{\text {Old }}$ ). Therefore, through the application of very simple algebraic operations, (ii) is equivalent to $(\alpha+\gamma+\delta) \beta>\alpha(\beta+\gamma)$, from which we obtain

$$
(\gamma+\delta) \beta>\alpha \gamma
$$

Notice also that $\gamma+\delta=S(a, b)^{\text {Old }}-S(a, b)^{\text {New }}$ and for lemma 3 we have $S(a, b)^{\text {Old }}-S(a, b)^{\text {New }}>0$, such that $\gamma+\delta>0$ always. We prove also that $\beta>\alpha$. In fact,

$$
K(a, b)^{N e w}=S(a, b)^{N e w}+\left(\sum_{i \in C(b P a)^{N e w}} k_{i}+\sum_{j \in C(b Q a)^{N e w}}\left(1-c_{j}(a, b)\right) k_{j}\right)
$$

such that, remembering that $f \in C(b Q a)^{N e w}$, we get that the quantity in between big parentheses is always positive. Therefore $K(a, b)^{\text {New }}>S(a, b)^{\text {New }}$ and consequently $\beta>\alpha$.

Coming back to condition (iii), two cases are possible:

- $\gamma \leq 0$ : in this case $(\gamma+\delta) \beta>0$ and $\alpha \gamma \leq 0$ (notice that $\alpha$ equals $S(a, b)$ after decreasing $\Delta_{f}$ and, by Lemma 3, $S(a, b)$ is always non-negative), such that (iii) holds;

- $\gamma>0$ : since $\beta>\alpha$ and $\alpha \geq 0$, (iv) holds if $\delta \geq 0$, which is always true.

Therefore, in any case (iii) is true and we can conclude that, when decreasing $\Delta_{f}, c(a, b)$ also decreases.

(c) Criterion $f$ belongs to $C(b Q a)$ and it remains in $C(b Q a)$ after decreasing $\Delta_{f}$.

This case is analogous to the previous one, because $c_{f}(a, b)$ changes its new value and becomes $c_{f}(a, b)-\Delta$, with $\Delta>0$. Thus, when decreasing $\Delta_{f}, c(a, b)$ also decreases.

(d) Criterion $f$ moves from $C(b Q a)$ to $C(b P a)$.

Let $\bar{C}(b P a)^{\text {Old }}, L(a, b)^{\text {Old }}$, and $O(a, b)^{\text {Old }}$ denote the sets $\bar{C}(b P a), L(a, b)$, and $O(a, b)$, respectively, before decreasing $\Delta_{f}$, and let $\bar{C}(b P a)^{\text {New }}, L(a, b)^{\text {New }}$, and $O(a, b)^{\text {New }}$ denote the same sets after decreasing $\Delta_{f}$. We have,

$$
\begin{aligned}
& \bar{C}(b P a)^{\text {New }}=\bar{C}(b P a)^{\text {Old }} \backslash\{f\} \\
& L(a, b)^{\text {New }}=L(a, b)^{\text {Old }} \backslash\left\{\{j, f\}: j \in \bar{C}(b P a)^{\text {Old }} \backslash\{f\}\right\} \\
& O(a, b)^{\text {New }}=O(a, b)^{\text {Old }} \backslash\left\{(f, h): h \in C(b P a)^{\text {Old }}\right\} \cup\left\{(i, f): i \in C(b P a)^{\text {Old }} \backslash\{f\}\right\}
\end{aligned}
$$

Therefore all the summations in the numerator and in the the denominatior of (6) are affected. Consider the following additional notation, 


$$
\begin{aligned}
& \alpha=S(a, b)^{\text {New }} \\
& \beta=K(a, b)^{\text {New }} \\
& \gamma=K(a, b)^{\text {Old }}-K(a, b)^{\text {New }} \\
& \delta=k_{f} c_{f}(a, b)
\end{aligned}
$$

where $K(a, b)^{\text {Old }}$ denotes the value of $K(a, b)$ before $\Delta_{f}$ decreases, and $S(a, b)^{\text {New }}$ and $K(a, b)^{\text {New }}$ denote the value of $S(a, b)$ and $K(a, b)$ after $\Delta_{f}$ decreases.

Thus, we have that

$$
c(a, b)^{\text {Old }}=\frac{\alpha+\gamma+\delta}{\beta+\gamma} \quad \text { and } \quad c(a, b)^{N e w}=\frac{\alpha}{\beta}
$$

such that from the monotonicity condition $c(a, b)^{\text {Old }}>c(a, b)^{\text {New }}$ becomes as follows,

$$
\frac{\alpha+\gamma+\delta}{\beta+\gamma}>\frac{\alpha}{\beta}
$$

Observe that $\beta>0$ and $\beta+\gamma>0$ (because $\beta=K(a, b)^{\text {New }}$ and $\beta+\gamma=K(a, b)^{\text {Old }}$ ). Therefore, through the application of very simple algebraic operations, (iv) is equivalent to $(\alpha+\gamma+\delta) \beta>\alpha(\beta+\gamma)$, from which we obtain

$$
(\gamma+\delta) \beta>\alpha \gamma
$$

Notice also that $\gamma+\delta=S(a, b)^{\text {Old }}-S(a, b)^{\text {New }}$ and for lemma 3 we have $S(a, b)^{\text {Old }}-S(a, b)^{\text {New }}>0$, such that $\gamma+\delta>0$ always. As proved in previous point (b), remember that $\beta>\alpha$.

Coming back to condition $(v)$, two cases are possible:

- $\gamma \leq 0$ : in this case $(\gamma+\delta) \beta>0$ and $\alpha \gamma \leq 0$ (notice that $\alpha$ equals $S(a, b)$ after decreasing $\Delta_{f}$ and, by Lemma 3, $S(a, b)$ is always non-negative), such that $(v)$ holds;

- $\gamma>0$ : since, as proved in previous point (b), $\beta>\alpha$, and $\alpha \geq 0$, (v) holds if $\delta \geq 0$, which is always true.

Therefore, in any case $(v)$ is true and we can conclude that, when decreasing $\Delta_{f}, c(a, b)$ also decreases. 


\section{References}

[1] C. Bana e Costa, J-M. De Corte, and J-C. Vansnick. On the mathematical foundations of MACBETH. In J. Figueira, S. Greco, and M. Ehrgott, editors, Multiple Criteria Decision Analysis: The State of the Art Surveys, pages 409-442. Springer Science+Business Media, Inc., New York.

[2] C. Bana e Costa and J. Vansnick. MACBETH - An interactive path towards the construction of cardinal value functions. International Transactions in Operational Research, 1:489-500, 1994.

[3] R. Bisdorff. Logical foundation of multicriteria preference aggregation. In D. Bouyssou, E. Jacquet-Lagrèze, P. Perny, R. Słowiński, D. Vanderpooten, and Ph. Vincke, editors, Aiding Decisions with Multiple Criteria: Essays in Honour of Bernard Roy, pages 379-403. Kluwer Academic Publishers, Dordrecht, 2001.

[4] G. Choquet. Theory of capacities. Annales de l'Institut Fourier, 5:131-295, 1953.

[5] W. Edwards. How to use multiple attribute utility measurement for social decision-making. IEEE Transactions on Systems, Man, and Cybernetics, SMC-7,5:326-340, 1977.

[6] J. Figueira, S. Greco, and M. Ehrgott, editors. Multiple Criteria Decision Analysis: The State of the Art Surveys. Springer Science+Business Media, Inc., New York, 2005.

[7] J. Figueira, V. Mousseau, and B. Roy. ELECTRE methods. In J. Figueira, S. Greco, and M. Ehrgott, editors, Multiple Criteria Decision Analysis: The State of the Art Surveys, pages 133-162. Springer Science+Business Media, Inc., New York, 2005.

[8] J. Figueira and B. Roy. Determining the weights of criteria in the ELECTRE type methods with a revised Simos' procedure. European Journal of Operational Research, 139:317-326, 2002.

[9] M. Grabisch. The application of fuzzy integrals in multicriteria decision making. European Journal of Operational Research, 89:445-456, 1996.

[10] M. Grabisch and C. Labreuche. Fuzzy measures and integrals in MCDA. In J. Figueira, S. Greco, and M. Ehrgott, editors, Multiple Criteria Decision Analysis: The State of the Art Surveys, pages 563-608. Springer Science+Business Media, Inc., New York.

[11] M. Grabisch and C. Labreuche. Bi-capacities - Part I: Definition, Möbius transform and interaction. Fuzzy Sets and Systems, 151:211-236, 2005.

[12] M. Grabisch and C. Labreuche. Bi-capacities - Part II: The Choquet integral. Fuzzy Sets and Systems, 151:237-259, 2005.

[13] S. Greco and J. Figueira. Dealing with interaction between bi-polar multiple criteria preferences in outranking methods. Research Report 11-2003, INESC-Coimbra, Portugal, 2003. [available at: http://www.inescc.pt/pubinter.php].

[14] S. Greco, B. Matarazzo, and R. Słowiński. Rough sets theory for multicriteria decision analysis. European Journal of Operational Research, 129:1-47, 2001.

[15] S. Greco, B. Matarazzo, and R. Słowiński. Bipolar Sugeno and Choquet integrals. In B. De Baets, J. Fodor, and G. Pasi, editors, Proceedings of the EURO Working Group on Fuzzy Sets, Workshop on Information Systems (EUROFUSE'2002), pages 191-196. Varenna, Italy, 2002.

[16] C. Hwang and K. Yoon. Multiple Attribute Decision Making: Methods and Applications - A State-of-the-Art Surveys. SpringerVerlag, Berlin, 1986.

[17] R. Keeney and H. Raiffa. Decisions with Multiple Objectives: Preferences and Value Tradeoffs. John Wiley \& Sons, New York, 1976.

[18] J-M. Martel and B. Roy. Analyse de la signifiance de diverses procédures d'agrégation multicritère. INFOR, 44(3):119-215, 2006.

[19] V. Mousseau. Problèmes liés à l'évaluation de l'importance relative des critères en aide multicritère à la décision : Réflexions théoriques, expérimentations et implémentations informatiques. PhD thesis, Université Paris-Dauphine, 1993.

[20] V. Mousseau. Eliciting information concerning the relative importance of criteria. In P. Pardalos, Y. Siskos, and C. Zopounidis, editors, Advances in Multicriteria Analysis, Nonconvex Optimization and its Applications, pages 17-43. Kluwer Academic Publishers, Dordrecht, 1995.

[21] G-C. Rota. On the foundations of combinatorial theory, I. Theory of Möbius inversion. Zeitschrift für Wahrscheinlichkeitstheorie, 2:340-368, 1964.

[22] B. Roy. The outranking approach and the foundations of ELECTRE methods. Theory and Decision, 31:49-73, 1991.

[23] B. Roy. Decision science or decision-aid science? European Journal of Operational Research, 66:184-203, 1993.

[24] B. Roy. Multicriteria Methodology for Decision Aiding. Nonconvex Optimization and its Applications. Kluwer Academic Publishers, Dordrecht, 1996.

[25] B. Roy. A propos de la signification des dépendances entre critères : Quelle place et quels modèles de prise en compte pour l'aide à la décision? 2007. Cahiers du LAMSADE 244, Université Paris-Dauphine, France. [To appear in RAIRO Operations Research / Recherche Opérationnelle].

[26] B. Roy and D. Bouyssou. Aide Multicritère à la Décision : Méthodes et Cas. Economica, Paris, 1993.

[27] B. Roy and V. Mousseau. A theoretical framework for analysing the notion of relative importance of criteria. Journal of Multi-Criteria Decision Analysis, 5:145-159, 1996.

[28] B. Roy and D. Vanderpooten. An overview on 'the European School of MCDA: Emergence, basic features and current works'. European Journal of Operational Research, 99(1):26-27, 1997.

[29] T. Saaty. The analytic hierarchy and analytic network processes for the measurement of intangible criteria and for decisionmaking. In J. Figueira, S. Greco, and M. Ehrgott, editors, Multiple Criteria Decision Analysis: The State of the Art Surveys, pages 345-407. Springer Science+Business Media, Inc., New York. 
[30] Ph. Vincke. Multicriteria Decision-Aid. John Wiley \& Sons, Chichester, 1992.

[31] D. Von Winterfeldt and W. Edwards. Decision Analysis and Behavioral Research. Cambridge University Press, Cambridge, 1986. 